\title{
Variation de la dimension relative en géométrie analytique $p$-adique
}

\author{
Antoine Ducros
}

\begin{abstract}
Let $k$ be a complete, non-Archimedean valued field (the trivial absolute value is allowed) and let $\varphi: X \rightarrow Y$ be a morphism between two Berkovich $k$-analytic spaces; we show that, for any integer $n$, the set of points of $X$ at which the local dimension of $\varphi$ is at least equal to $n$ is a Zariski-closed subset of $X$. In order to establish it, we first prove an analytic analogue of Zariski's Main Theorem, and we also introduce, and study, the notion of an analytic system of parameters at a point.
\end{abstract}

\section{Introduction}

Soit $f: \mathscr{Y} \rightarrow \mathscr{X}$ un morphisme localement de présentation finie entre schémas. Pour tout point $y$ de $\mathscr{Y}, \operatorname{notons} \operatorname{dim}_{y} f$ la dimension relative de $f$ en $y$, autrement dit la dimension de la fibre $f^{-1}(f(y))$ au voisinage de $y$. Il est bien connu que la fonction $y \mapsto \operatorname{dim}_{y} f$ est semi-continue supérieurement, c'est-à-dire que pour tout entier $n$, le sous-ensemble $\mathscr{D}_{n}(f)$ de $\mathscr{Y}$ formé des $y$ tels que $\operatorname{dim}_{y} f \geqslant n$ est un fermé de Zariski.

Dans ce texte, nous démontrons l'assertion analogue en géométrie analytique au sens de Berkovich (théorème 4.9): si $k$ est un corps ultramétrique complet et si $\varphi: Y \rightarrow X$ est un morphisme d'espaces $k$-analytiques, alors pour tout entier $n$, le sous-ensemble $\mathscr{D}_{n}(\varphi)$ de $Y$ est un fermé de Zariski de $Y$.

Le théorème correspondant en géométrie analytique rigide a été démontré par Kiehl [Kie68, 3.7], par des techniques d'algèbre commutative. La première idée qui se présente à l'esprit pour prouver l'énoncé que nous avons en vue consiste à se ramener à ce théorème de Kiehl par changement du corps de base; elle n'aboutit malheureusement pas, pour une raison simple: les méthodes de Kiehl ne garantissent pas a priori que la formation de l'ensemble analytique $\mathscr{D}_{n}(\varphi)$, dans le contexte de la géométrie rigide, commute aux changements de corps de base.

Il est néanmoins possible d'utiliser ce qu'a fait Kiehl pour établir notre théorème, mais d'une manière absolument non triviale, requérant un travail préparatoire délicat; cela a été réalisé par Berkovich, dans des notes non publiées. Mentionnons à ce propos que dans un article récent [Con06], Conrad se fonde sur ce résultat de Berkovich... précisément pour démontrer que la formation de $\mathscr{D}_{n}(\varphi)$ en géométrie rigide commute aux changements de corps de base!

Nos méthodes sont complètement diffèrentes de celles de Berkovich, et ne font pas appel à l'article de Kiehl; elles sont inspirées par l'étude que Raynaud et Gruson ont consacrée à la platitude et la platification [RG71]. Nous espérons ainsi utiliser ultérieurement les résultats intermédiaires obtenus ici pour mieux comprendre ces notions dans le contexte analytique; c'est l'une des motivations du présent travail.

Received 30 March 2006, accepted in final form 16 July 2007, published online 9 November 2007. 2000 Mathematics Subject Classification 14G22, $14 \mathrm{~A} 99$.

Keywords: Berkovich analytic spaces, dimension theory.

This journal is (c) Foundation Compositio Mathematica 2007. 


\section{A. Ducros}

\section{Les préliminaires techniques}

Faute de références disponibles dans la littérature, nous entamons cet article par une section dévolue à l'étude de la notion de dimension en géométrie analytique à la Berkovich; nous établissons à son sujet les résultats de base, qui n'ont pour l'essentiel rien de surprenant: tout ce qu'on espère vrai l'est effectivement.

Nous prouvons ensuite un résultat nouveau (théorème 2.7), qui joue un rôle crucial au cours de cet article, et qui peut par ailleurs présenter un intérêt intrinsèque : il s'agit d'une forme de Nullstellensatz pour les algèbres affinoïdes au sens de Berkovich, plus générales que celles que considérait Tate (Berkovich accepte en effet les polydisques fermés de polyrayon arbitraire). Plus précisément, nous donnons une description exhaustive des algèbres affinoïdes sur un corps $k$ fixé qui sont des corps ; ce ne sont pas nécessairement des extensions finies de $k$, comme le montre l'exemple de l'anneau des fonctions analytiques de la couronne $|T|=r$, où $r \notin \sqrt{\left|k^{*}\right|}$.

\section{Les théorèmes géométriques}

$\mathrm{Au} \S 3$, nous commençons par établir (théorème 3.2) un analogue analytique du Main Theorem de Zariski; il s'agit de donner une description d'un morphisme au voisinage d'un point en lequel il est de dimension nulle. Nous y parvenons à l'aide du cas sans bord, dû à Berkovich [Ber93, Proposition 3.1.4], et de la théorie de la « réduction des germes d'espaces analytiques » qu'a développée Temkin dans [Tem04].

Nous montrons ensuite (proposition 3.5) que le lieu des points en lesquels un morphisme donné $\varphi: Y \rightarrow X$ est de dimension nulle est un ouvert de Zariski de $Y$. On procède comme suit. Tout d'abord, on peut faire l'hypothèse que le lieu en question est non vide, que $X$ et $Y$ sont affinoïdes et que $Y$ est réduit; grâce à notre « Main Theorem », on exhibe une factorisation $Y \rightarrow Y^{b} \rightarrow X$ de $\varphi$, où $Y \rightarrow Y^{b}$ est fini, radiciel et surjectif, et où $\Omega_{Y^{b} / X}$ s'annule en au moins un point de $Y^{b}$. Il existe par conséquent un ouvert de Zariski non vide $Z^{b}$ de $Y^{b}$ tel que $\Omega_{Z^{b} / X}$ soit trivial ; la dimension de $Z^{b} \rightarrow X$ est donc identiquement nulle. Si l'on désigne par $Z$ l'image réciproque de $Z^{\mathrm{b}}$ sur $Y$, alors la dimension de $\varphi_{\mid Z}$ est identiquement nulle. Soit $F$ le complémentaire de $Z$ dans $Y$. Par récurrence noethérienne, l'ensemble des $y$ appartenant à $F$ en lesquels la dimension de $\varphi_{\mid F}$ est nulle est un ouvert de Zariski $U$ de $F$. Comme la dimension de $\varphi_{\mid Z}$ est identiquement nulle on a équivalence, pour tout $y$ appartenant à $F$, entre la nullité $\operatorname{de} \operatorname{dim}_{y} \varphi_{\mid F}$ et celle de $\operatorname{dim}_{y} \varphi$; dès lors l'ensemble des points $y$ de $Y$ tels que $\operatorname{dim}_{y} \varphi=0$ est exactement la réunion de $Z$ et $U$, et est donc un ouvert de Zariski de $Y$.

$\mathrm{Au}$ début de la section 4, nous introduisons un avatar analytique de la notion bien connue de «système de paramètres » et établissons un lemme technique à son sujet (lemme 4.5). Ce lemme et la proposition 3.5 sont utilisés pour prouver le théorème suivant (théorème 4.6) : soit $\varphi: Y \rightarrow X$ un morphisme entre espaces affinö̈des et soit $y$ un point de $Y$; notons $n$ la dimension de $\varphi$ en $y$. On peut alors factoriser $\varphi$ par une flèche $\psi$ de $Y$ vers $\mathbb{A}_{X}^{n}$ dont la dimension en y est nulle.

En combinant cette assertion et le «Main Theorem », on obtient (corollaire 4.7) l'existence localement sur la source d'une factorisation agréable d'un morphisme entre bons espaces analytiques ; c'est le pendant analytique d'un théorème de Raynaud et Gruson sur les morphismes de schémas [RG71, théorème 1.1.1]. Signalons ( $c f$. la remarque 4.8) que dans le cas rigide-analytique classique, Bosch et Lütkebohmert ont établi, via la géométrie formelle, l'existence d'une telle factorisation G-localement sur la source [BL93, théorème1.5].

Le théorème principal de notre article (théorème 4.9) s'obtient pour finir sans difficulté, en combinant le théorème 4.6 et la proposition 3.5 . 


\section{VARIATION DE LA DIMENSION RELATIVE}

\section{Remarques}

(1) Signalons une complication, inexistante dans le monde des schémas, qui intervient lorsqu'on veut démontrer le théorème 4.6 et impose certaines contorsions (dont le recours à la notion de «partie presque dense d'une algèbre de Banach », $c f$. définition 4.4) : si $x$ est un point de $X$ et si $Y_{x}$ désigne la fibre correspondante de $Y \rightarrow X$, la topologie de Zariski sur $Y_{x}$ est en général strictement plus fine que celle induite par la topologie de Zariski de $Y$.

(2) Nous avons choisi d'inclure le cas des corps à valeur absolue triviale, bien qu'il requière par endroits un traitement spécifique alourdissant un peu la rédaction. Mais il nous semblait important de le prendre en compte: la géométrie analytique sur ce type de corps a d'ores et déjà montré son utilité [Ber96, Thu07, Ber08]; elle joue de surcroît un rôle majeur dans la théorie des espaces de Berkovich sur un anneau d'entiers de corps de nombres, que Jérôme Poineau développe actuellement dans sa thèse.

\section{Conventions}

0.1 Dans ce texte, sauf mention expresse du contraire, les normes d'algèbres seront toujours sous-multiplicatives, les morphismes d'algèbres normées seront bornés, et les structures d'algèbres normées seront considérées à équivalence près.

0.2 Un corps ultramétrique complet est un corps muni d'une valeur absolue ultramétrique pour laquelle il est complet; le corps résiduel d'un corps ultramétrique complet $K$ est noté $\widetilde{K}$.

0.3 Une extension complète d'un corps ultramétrique complet $K$ est un corps ultramétrique complet $L$ muni d'une injection isométrique $K \hookrightarrow L$; une telle extension étant donnée, on pose

$$
\mathrm{d}(L / K)=\operatorname{deg} \operatorname{tr}(\widetilde{L} / \widetilde{K})+\operatorname{dim}_{\mathbb{Q}} \mathbb{Q} \otimes_{\mathbb{Z}}\left(\left|L^{*}\right| /\left|K^{*}\right|\right) \in \mathbb{N} \cup\{+\infty\} .
$$

On fixe pour toute la suite du texte un corps ultramétrique complet $k$; sa valeur absolue peut être triviale; on note $p$ son exposant caractéristique.

0.4 Dans cet article, la notion d'espace analytique est à prendre au sens de Berkovich [Ber90, Ber93] ; les notions d'algèbre $k$-affinö̈de et d'algèbre strictement $k$-affinoïde sont celles du chapitre 2 de [Ber90]. Si $X$ est un espace $k$-analytique, le corps résiduel complété d'un point $x$ de $X$ sera noté $\mathscr{H}(x)$; c'est la plus petite extension complète de $k$ dans laquelle vivent les évaluations des fonctions analytiques en $x$. Un point $x$ de $X$ est dit rigide si $\mathscr{H}(x)$ est une extension finie de $k$.

$0.5 \mathrm{Si} \mathscr{A}$ (respectivement $X$ ) est une algèbre $k$-affinoïde (respectivement un espace $k$-analytique) et si $L$ est une extension complète de $k$, l'algèbre $L$-affinö̈de $L \widehat{\otimes}_{k} \mathscr{A}$ (respectivement l'espace $L$ analytique $X \times_{k} L$ ) est noté(e) $\mathscr{A}_{L}$ (respectivement $X_{L}$ ). L'application naturelle $X_{L} \rightarrow X$ est surjective: sa fibre en un point $x$ de $X$ s'identifie en effet à $\mathscr{M}\left(\mathscr{H}(x) \widehat{\otimes}_{k} L\right)$; or $\mathscr{H}(x) \otimes_{k} L$ s'injecte dans $\mathscr{H}(x) \widehat{\otimes}_{k} L$ (cf. [Gru66, théorème $1,4^{\circ}$ ]) qui est donc non nul; on conclut à l'aide du théorème 1.2.1 de [Ber90]. On dit que $L$ déploie $\mathscr{A}$ (respectivement $X$ ) si $\left|L^{*}\right| \neq\{1\}$ et si $\mathscr{A}_{L}$ (respectivement $X_{L}$ ) est strictement $L$-affinoïde (respectivement strictement $L$-analytique).

0.6 Soit $\mathscr{A}$ une algèbre $k$-affinoïde, soit $X$ l'espace $\mathscr{M}(\mathscr{A})$ et soit $\mathscr{X}$ le spectre de $\mathscr{A}$; le schéma $\mathscr{X}$ est noethérien [Ber90, proposition 2.1.3]. L'application continue naturelle $\rho: X \rightarrow \mathscr{X}$ est surjective [Ber93, proposition 2.1.1]. On appelle topologie de Zariski de $X$ la topologie dont les fermés sont les images réciproques de fermés de Zariski de $\mathscr{X}$; l'application $\mathscr{F} \mapsto \rho^{-1}(\mathscr{F})$ établit, en vertu de la surjectivité de $\rho$, une bijection entre l'ensemble des fermés de Zariski de $\mathscr{X}$ et celui des fermés 


\section{A. Ducros}

de Zariski de $X$, dont la réciproque est $\mathscr{G} \mapsto \rho(\mathscr{G})$. Les notions de composante irréductible, de dimension de Krull, de codimension de Krull, appliquées à $X$ ou à certains de ses fermés de Zariski, seront toujours relatives à la topologie de Zariski.

Un sous-ensemble $Y$ de $X$ est un fermé de Zariski si et seulement si il peut être défini comme le lieu des zéros d'un idéal $\mathscr{I}$ de $\mathscr{A}$. En général, un tel $\mathscr{I}$ n'est pas unique; seul $\sqrt{\mathscr{I}}$ est canoniquement déterminé, c'est l'ensemble des fonctions s'annulant en tout point de $Y$. Chaque choix d'un $\mathscr{I}$ convenable permet de munir $Y$ d'une structure d'espace $k$-affinoïde, à savoir celle associée au quotient $\mathscr{A} / \mathscr{I}$. Il arrivera souvent, lorsque cela ne prête pas à conséquence, que l'on considère implicitement un fermé de Zariski de $X$ comme un espace affinoïde sans prendre la peine de préciser la structure pour laquelle on a opté.

Si $Y$ est un fermé de Zariski de $X$ et si $L$ est une extension complète de $k$, l'image réciproque de $Y$ sur sur $X_{L}$ est un fermé de Zariski que l'on désigne par $Y_{L}$; cette notation est compatible avec l'abus qui consiste à considérer indifféremment $Y$ comme un simple sous-ensemble de $X$ ou comme un espace $k$-affinö̈de. Si $Y$ et $Z$ sont deux fermés de Zariski distincts de $X$, alors $Y_{L} \neq Z_{L}$, puisque $Y_{L} \rightarrow Y$ et $Z_{L} \rightarrow Z$ sont surjectifs.

0.7 Si $X$ est un espace $k$-affinoïde et si $\left(X_{i}\right)$ est un redouvrement fini de $X$ par des domaines affinoïdes, une partie $Y$ de $X$ telle que $Y \cap X_{i}$ soit pour tout $i$ un fermé de Zariski de $X_{i}$ est un fermé de Zariski de $X$ (ceci découle du théorème de Kiehl, cf. [Ber93, §1.2]). Si $X$ est un espace $k$ analytique, on dira qu'une partie $Y$ de $X$ est un fermé de Zariski de $X$ s'il existe un G-recouvrement $\left(X_{i}\right)$ de $X$ par des domaines affinoïdes tel que $Y \cap X_{i}$ soit pour tout $i$ un fermé de Zariski de $X_{i}$; la remarque qui précède assure que cette définition est compatible avec celle déjà énoncée pour les espaces affinoïdes.

0.8 Si $\mathscr{A}$ est une algèbre $k$-affinoïde, une $\mathscr{A}$-algèbre de Banach finie est une $\mathscr{A}$-algèbre de Banach $\mathscr{B}$ qui est isomorphe, en tant que $\mathscr{A}$-module de Banach, à un quotient d'une somme directe finie de copies de $\mathscr{A}$, munie de la norme du maximum. En s'appuyant notamment sur les résultats du paragraphe 3.7.2 de [BGR84], Berkovich a prouvé les faits énoncés ci-dessous [Ber90, propositions 2.1.9, 2.1.10 et 2.1 .12$].^{1}$

Un morphisme de $\mathscr{A}$-algèbres entre deux $\mathscr{A}$-algèbres de Banach finies est automatiquement borné, et même admissible. Si $\mathscr{B}$ est une $\mathscr{A}$-algèbre finie, elle admet une unique structure de $\mathscr{A}$-algèbre de Banach finie, qui en fait une algèbre $k$-affinoïde (pour ce dernier point, appliquer la proposition 2.1.11 et le corollaire 2.1.8 de [Ber90] pour se ramener à la proposition 4 de [BGR84, 6.1.3]).

Un morphisme $\mathscr{M}(\mathscr{B}) \rightarrow \mathscr{M}(\mathscr{A})$ entre espaces $k$-affinoïdes sera dit fini si $\mathscr{B}$ est une $\mathscr{A}$-algèbre de Banach finie. Sous cette hypothèse, si $\mathscr{C}$ est une algèbre affinoïde sur une extension complète $L$ de $k$, alors $\mathscr{C} \otimes \mathscr{A} \mathscr{B} \rightarrow \mathscr{C} \widehat{\otimes} \mathscr{A} \mathscr{B}$ est bijective, et $\mathscr{C} \widehat{\otimes} \mathscr{A} \mathscr{B}$ est une $\mathscr{C}$-algèbre de Banach finie; en particulier, si $x \in \mathscr{M}(\mathscr{A})$, alors $\mathscr{H}(x) \widehat{\otimes} \mathscr{A} \mathscr{B}=\mathscr{H}(x) \otimes_{\mathscr{A}} \mathscr{B}$, et l'image réciproque de $x$ sur $\mathscr{M}(\mathscr{B})$ est ainsi non vide si et seulement si $\mathscr{B} \otimes \mathscr{A} \mathscr{H}(x)$ est non nul. Il s'ensuit qu'un morphisme fini entre espaces $k$-affinoïdes qui est induit par un morphisme injectif d'algèbres $k$-affinoïdes est surjectif.

Si $\left|k^{*}\right| \neq\{1\}$, toute $k$-algèbre normée finie est une $k$-algèbre de Banach finie par l'équivalence des normes en dimension finie; si $\left|k^{*}\right|=\{1\}$, une $k$-algèbre de Banach finie est une $k$-algèbre finie munie d'une norme équivalente à la norme triviale. On en déduit que si $L$ est une extension finie de $k$, sa structure de $k$-algèbre de Banach finie peut toujours être définie par l'unique prolongement à $L$ de la valeur absolue de $k$.

\footnotetext{
${ }^{1}$ Dans la démonstration de la proposition 2.1.9 de [Ber90], la justification du fait que Ker $\pi$ est fermé est erronée ; on peut la remplacer par un raisonnement analogue à celui de la preuve de la proposition 2.1.3 de [Ber90], combiné avec la proposition 1 de [BGR84, 3.7.2].
} 


\section{VARIATION DE LA DIMENSION RELATIVE}

\section{Les polyrayons}

\section{Théorie de la dimension}

1.1 Un polyrayon est une famille finie $\left(r_{1}, \ldots, r_{l}\right)$ de réels strictement positifs; l'entier $l$ est la longueur du polyrayon. On dit que $\left(r_{1}, \ldots, r_{l}\right)$ est $k$-libre si $\left(r_{1}, \ldots, r_{l}\right)$ est libre lorsqu'on la voit comme une famille d'éléments du $\mathbb{Q}$-espace vectoriel $\mathbb{Q} \otimes_{\mathbb{Z}}\left(\mathbb{R}_{+}^{*} /\left|k^{*}\right|\right)$.

1.2 Soit $\mathbf{r}=\left(r_{1}, \ldots, r_{l}\right)$ un polyrayon. On note $k_{\mathbf{r}}$ la $k$-algèbre de Banach définie comme l'ensemble des sommes formelles $\sum_{\mathbb{Z}^{l}} a_{\mathbf{I}} \mathbf{T}^{\mathbf{I}}$ où $\mathbf{T}$ est un $l$-uplet d'indéterminées et où les $a_{\mathbf{I}}$ sont des éléments de $k$ tels que $\left|a_{\mathbf{I}}\right| \mathbf{r}^{\mathbf{I}}$ tende vers zéro quand $|\mathbf{I}|$ tend vers l'infini; les deux opérations de $\mathscr{A}_{\mathbf{r}}$ sont celles que l'on imagine, sa norme est l'application $\sum a_{\mathbf{I}} \mathbf{T}^{\mathbf{I}} \mapsto \max \left|a_{\mathbf{I}}\right| \mathbf{r}^{\mathbf{I}}$. L'algèbre $k_{\mathbf{r}}$ est $k$-affinoïde [Ber90, § 2.1].

1.2.1 La norme de $k_{\mathrm{r}}$ est multiplicative. Il est en effet clair qu'elle envoie 1 sur 1 . Par ailleurs, considérons deux éléments non nuls $A=\sum a_{\mathbf{I}} \mathbf{T}^{\mathbf{I}}$ et $B=\sum b_{\mathbf{I}} \mathbf{T}^{\mathbf{I}}$ de $k_{\mathbf{r}} ;$ munissons $\mathbb{Z}^{l}$ de l'orde lexicographique, et soit $\mathbf{U}$ (respectivement $\mathbf{V}$ ) le plus grand des indices $\mathbf{I}$ tels que $\left|a_{\mathbf{I}}\right| \mathbf{r}^{\mathbf{I}}=\|A\|$ (respectivement $\left|b_{\mathbf{I}}\right| \mathbf{r}^{\mathbf{I}}=\|B\|$ ). Le coefficient $c$ de $\mathbf{T}^{\mathbf{U}+\mathbf{V}}$ dans la série produit $A B$ est alors somme de $a_{\mathbf{U}} b_{\mathbf{V}}$ et de termes de la forme $a_{\mathbf{I}} b_{\mathbf{J}}$ qui, par construction, sont tels que $\left|a_{\mathbf{I}} b_{\mathbf{J}}\right| \mathbf{r}^{\mathbf{U}+\mathbf{V}}<\left|a_{\mathbf{U}} b_{\mathbf{V}}\right| \mathbf{r}^{\mathbf{U}+\mathbf{V}}$. On en déduit que $\|A\| \cdot\|B\| \geqslant\|A B\| \geqslant|c| \mathbf{r}^{\mathbf{U}+\mathbf{V}}=\left|a_{\mathbf{U}}\right| \mathbf{r}^{\mathbf{U}}\left|b_{\mathbf{V}}\right| \mathbf{r}^{\mathbf{V}}=\|A\| \cdot\|B\|$, d'où l'assertion. Remarquons que celle-ci implique entre autres que $k_{\mathrm{r}}$ est intègre.

1.2.2 Supposons que $\mathbf{r}$ est $k$-libre. Dans ce cas, $k_{\mathbf{r}}$ est un corps. En effet, soit $A=\sum a_{\mathbf{I}} \mathbf{T}^{\mathbf{I}}$ un élément non nul de $k_{\mathbf{r}}$. Comme $\mathbf{r}$ est $k$-libre, si $\mathbf{I}$ et $\mathbf{J}$ sont deux indices distincts tels que $a_{\mathbf{I}} \neq 0$ et $b_{\mathbf{I}} \neq 0$, alors $\left|a_{\mathbf{I}}\right| \mathbf{r}^{\mathbf{I}} \neq\left|b_{\mathbf{J}}\right| \mathbf{r}^{\mathbf{J}}$; on en déduit qu'il existe un unique indice $\mathbf{U}$ tel que $\left|a_{\mathbf{U}}\right| \mathbf{r}^{\mathbf{U}}=\|A\|$. On peut en conséquence écrire $A=a_{\mathbf{U}} \mathbf{T}^{\mathbf{U}}(1+B)$, avec $\|B\|<1$; dès lors, $A$ est inversible.

La norme de $k_{\mathbf{r}}$ étant multiplicative, c'est une valeur absolue qui fait de $k_{\mathbf{r}}$ une extension complète de $k$. Il est immédiat que $\left|k_{\mathbf{r}}^{*}\right|=\left|k^{*}\right| \oplus r_{1}^{\mathbb{Z}} \oplus \cdots \oplus r_{l}^{\mathbb{Z}}$. D'après ce qui précède, un élément de $k_{\mathbf{r}}^{*}$ est de valeur absolue égale à 1 si et seulement il s'écrit $a_{\mathbf{U}}(1+B)$ avec $a_{\mathbf{U}} \in k^{\mathrm{o}}-k^{\mathrm{oo}}$ et $\|B\|<1$; on en conclut que $\widetilde{k_{\mathbf{r}}}=\widetilde{k}$, et que $\mathrm{d}\left(k_{\mathbf{r}} / k\right)=l$.

1.2.3 Si $\mathbf{r}$ est un polyrayon et si $\mathscr{A}$ (respectivement $X$ ) est une algèbre $k$-affinoïde (respectivement un espace $k$-analytique) on posera $\mathscr{A}_{\mathbf{r}}=k_{\mathbf{r}} \widehat{\otimes}_{k} \mathscr{A}$ (respectivement $\left.X_{\mathbf{r}}=X \times_{k} \mathscr{M}\left(k_{\mathbf{r}}\right)\right)$. On dira que $\mathbf{r}$ déploie $\mathscr{A}$ (respectivement $X)$ s'il est $k$-libre et si $k_{\mathrm{r}}$ déploie $\mathscr{A}$ (respectivement $\left.X\right)$.

Lemme 1.3. Soit $\mathscr{A}$ une algèbre $k$-affinoïde et soit $\mathbf{r}$ un polyrayon. L'algèbre $\mathscr{A}_{\mathbf{r}}$ est réduite (respectivement intègre) si et seulement si $\mathscr{A}$ est réduite (respectivement intègre).

Démonstration. Comme $\mathscr{A}$ s'injecte dans $\mathscr{A}_{\mathbf{r}}$, elle est réduite (respectivement intègre) dès que $\mathscr{A}_{\mathbf{r}}$ est réduite (respectivement intègre). Établissons les implications réciproques, en supposant pour commencer que $\mathscr{A}$ réduite. Soit $A=\sum a_{\mathbf{I}} \mathbf{T}^{\mathbf{I}}$ un élément nilpotent de $\mathscr{A}_{\mathbf{r}}$. Soit $x$ un point appartenant à $\mathscr{M}(\mathscr{A})$. L'élément $\sum a_{\mathbf{I}}(x) \mathbf{T}^{\mathbf{I}}$ de $\mathscr{H}(x)_{\mathbf{r}}$ est nilpotent, donc nul puisque $\mathscr{H}(x)_{\mathbf{r}}$ est intègre d'après le 1.2 .1 ci-dessus. En conséquence, chacun des $a_{\mathbf{I}}(x)$ est nul. Pour tout $\mathbf{I}$, l'élément $a_{\mathbf{I}}$ de $\mathscr{A}$ s'annule ainsi en chaque point de $\mathscr{M}(\mathscr{A})$, et est de ce fait nilpotent. Comme $\mathscr{A}$ est réduite, tous les $a_{\mathbf{I}}$ sont nuls et $A=0$.

Supposons maintenant $\mathscr{A}$ intègre. Elle est alors non nulle, et l'on en déduit immédiatement que $\mathscr{A}_{\mathbf{r}}$ est non nulle. Soient $A=\sum a_{\mathbf{I}} \mathbf{T}^{\mathbf{I}}$ et $B=\sum b_{\mathbf{I}} \mathbf{T}^{\mathbf{I}}$ deux éléments de $\mathscr{A}_{\mathbf{r}}$ de produit nul, et soit $\mathscr{I}_{A}$ (respectivement $\mathscr{I}_{B}$ ) l'idéal de $\mathscr{A}$ engendré par les $a_{\mathbf{I}}$ (respectivement les $b_{\mathbf{I}}$ ). Pour tout point $x$ de $\mathscr{M}(\mathscr{A})$, l'intégrité de $\mathscr{H}(x)_{\mathbf{r}}$ implique que $\sum a_{\mathbf{I}}(x) \mathbf{T}^{\mathbf{I}}=0$ ou $\sum b_{\mathbf{I}}(x) \mathbf{T}^{\mathbf{I}}=0$, autrement dit que ou bien tous les $a_{\mathbf{I}}(x)$ sont nuls, ou bien que tous les $b_{\mathbf{I}}(x)$ sont nuls. L'espace $\mathscr{M}(\mathscr{A})$ est donc 


\section{A. Ducros}

réunion des deux fermés de Zariski respectivement définis par $\mathscr{I}_{A}$ et $\mathscr{I}_{B}$; comme $\mathscr{A}$ est intègre, l'un de ces deux idéaux est réduit à $\{0\}$, et la série correspondante est nulle.

1.4 Soit $\mathscr{A}$ une algèbre $k$-affinoïde et soit $X$ l'espace $\mathscr{M}(\mathscr{A})$. Il résulte du théorème 2.2 .1 de [Ber93] que $X$ est réduit en tant qu'espace localement annelé si et seulement si $\mathscr{A}$ est réduite; on dira alors tout simplement que $X$ est réduit, et on le qualifiera d'intègre s'il est réduit et irréductible, autrement dit si $\mathscr{A}$ est intègre. Si $X$ est réduit et si $Y$ est un domaine affinoïde de $X$, alors $Y$ est réduit. Pour le voir, on se ramène grâce au lemme 1.3 au cas où $\left|k^{*}\right|$ n'est pas triviale et où $X$ et $Y$ sont strictement $k$-affinoïdes. Soit $\mathscr{B}$ l'algèbre associée à $Y$. Il suffit de montrer que pour tout idéal maximal $\mathfrak{m}$ de $\mathscr{B}$, l'anneau local $\mathscr{B}_{\mathfrak{m}}$ est réduit; par le Nullstellensatz rigide-analytique et le théorème 2.2.8 de [Ber93], ceci revient à montrer que l'espace localement annelé $Y$ est réduit en chacun de ses points rigides. Or un tel point appartient toujours à l'intérieur topologique de $Y$ dans $X$, d'où l'assertion.

\section{La dimension $k$-analytique}

C'est une notion due à Berkovich [Ber90, § 2]; nous allons en rappeler la définition, et en indiquer quelques propriétés essentielles.

1.5 Soit $\mathscr{A}$ une algèbre strictement $k$-affinoïde non nulle. Le lemme de normalisation de Noether (algébrique si $\left|k^{*}\right|=\{1\}$, analytique sinon - cf. [BGR84, 6.1.2, corollaire 2]) assure l'existence d'un entier $n$ et d'une injection finie admissible $k\left\{T_{1}, \ldots, T_{n}\right\} \hookrightarrow \mathscr{A}$. La dimension de Krull de $k\left\{T_{1}, \ldots, T_{n}\right\}$ est égale à $n$ ([BGR84,6.1.2], remarque suivant le corollaire 2 ); il en va donc de même de celle de $\mathscr{A}$. Soit $L$ une extension complète de $k$. La flèche $L\left\{T_{1}, \ldots, T_{n}\right\} \rightarrow \mathscr{A}_{L}$ est une injection finie et admissible ([Gru66, théorème 1, $1^{\circ}$ ] et [Ber93, preuve du lemme 2.1.2]) ; en conséquence, la dimension de Krull de $\mathscr{A}_{L}$ est égale à $n$, donc à celle de $\mathscr{A}$.

DÉfinition 1.6 (Berkovich [Ber90, $\S 2]$ ). Soit $\mathscr{A}$ une algèbre $k$-affinoïde et soit $L$ une extension complète de $k$ déployant $\mathscr{A}$. Il résulte de ce qui précède que la dimension de Krull de $\mathscr{A}_{L}$ ne dépend pas de $L$; on l'appelle la dimension $k$-analytique de $\mathscr{A}$ et on la note $\operatorname{dim}_{k} \mathscr{A}$. Si $F$ est une extension complète de $k$ et si $L$ est une extension complète de $F$ qui déploie $\mathscr{A}_{F}$, alors $L$ déploie $\mathscr{A}$; on en déduit l'égalité $\operatorname{dim}_{k} \mathscr{A}=\operatorname{dim}_{F} \mathscr{A}_{F}$.

Lemme 1.7. Soit $\mathscr{A}$ une algèbre $k$-affinoïde. On a $\operatorname{dim}_{k} \mathscr{A}=0$ si et seulement si $\mathscr{A}$ est finie sur $k$, et $\mathscr{A}$ est alors une $k$-algèbre de Banach finie.

Démonstration. Soit $\mathbf{r}$ un polyrayon déployant $\mathscr{A}$.

Supposons que $\mathscr{A}$ est finie sur $k$. Choisissons une surjection admissible $k\left\{s_{1}^{-1} T_{1}, \ldots, s_{n}^{-1} T_{n}\right\} \rightarrow$ $\mathscr{A}$ (la notation $k\left\{s_{1}^{-1} T_{1}, \ldots, s_{n}^{-1} T^{n}\right\}$ est celle de [Ber90, $\left.\S 2\right]$ ). Comme $\mathscr{A}$ est finie sur $k$, l'image de chacun des $T_{i}$ est entière sur $k$; l'algèbre $\mathscr{A}_{\mathbf{r}}$ est topologiquement engendrée par les $1 \otimes T_{i}$, et donc par une $k_{\mathrm{r}}$-algèbre normée $\mathscr{B}$ de dimension finie. Puisque la valeur absolue de $k_{\mathrm{r}}$ n'est pas triviale, $\mathscr{B}$ est complète; elle coïncide par conséquent avec $\mathscr{A}_{\mathbf{r}}$, et dès $\operatorname{lors}_{\operatorname{dim}} \mathscr{A}=\operatorname{dim}_{\text {Krull }} \mathscr{A}_{\mathbf{r}}=0$.

Supposons que $\operatorname{dim}_{k} \mathscr{A}=0$. On a alors $\operatorname{dim}_{\text {Krull }} \mathscr{A}_{\mathrm{r}}=0$; le lemme de normalisation de Noether garantit que $\mathscr{A}_{\mathbf{r}}$ est finie sur $k_{\mathbf{r}} ;$ comme $\left|k_{\mathbf{r}}^{*}\right| \neq\{1\}$, l'algèbre $\mathscr{A}_{\mathbf{r}}$ est nécessairement une $k_{\mathbf{r}}$-algèbre de Banach finie; il découle alors de la proposition 2.1.11 de [Ber90] que $\mathscr{A}$ est une $k$-algèbre de Banach finie.

1.8 Si $X$ est un espace $k$-affinoïde, on appelle dimension $k$-analytique de $X$, et l'on note $\operatorname{dim}_{k} X$, la dimension $k$-analytique de l'algèbre associée; on a $\operatorname{dim}_{L} X_{L}=\operatorname{dim}_{k} X$ pour toute extension complète $L$ de $k$, la propriété correspondante au niveau des algèbres ayant été établie à la définition 1.6. 


\section{VARIATION DE LA DIMENSION RELATIVE}

Remarque 1.9. Soit $\mathscr{A}$ une algèbre $k$-affinoïde et soit $\mathscr{I}$ un idéal nilpotent de $\mathscr{A}$. On vérifie immédiatement que $\operatorname{dim}_{k} \mathscr{A}=\operatorname{dim}_{k} \mathscr{A} / \mathscr{I}$. Pour cette raison, on s'autorisera à parler de la dimension $k$-analytique d'un fermé de Zariski d'un espace affinoïde donné, sans préciser la structure dont on munit le fermé en question.

Lemme 1.10. Si $X$ est un espace $k$-affinoïde, $\operatorname{dim}_{k} X=0$ si et seulement si $X$ est constitué d'un ensemble fini de points rigides.

Démonstration. On peut supposer $X$ réduit; soit $\mathscr{A}$ l'algèbre $k$-affinoïde associée.

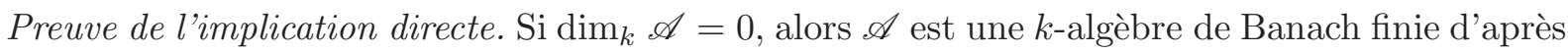
le lemme 1.7 ; c'est donc un produit $k_{1} \times \cdots \times k_{n}$ d'extensions finies de $k$, où chaque $k_{i}$ est munie de son unique valeur absolue qui prolonge celle de $k$; pour tout $i$, l'espace $\mathscr{M}\left(k_{i}\right)$ est constitué d'un point de corps résiduel complété égal à $k_{i}$, et l'implication directe est établie.

Preuve de la réciproque. Supposons que $X$ est constitué d'un ensemble fini $\left\{x_{1}, \ldots, x_{n}\right\}$ de points rigides. Comme $X$ est réduit, l'algèbre $\mathscr{A}$ s'injecte dans $\prod \mathscr{H}\left(x_{i}\right)$, qui est de dimension finie sur $k$. En conséquence, $\mathscr{A}$ est une $k$-algèbre finie, donc de dimension $k$-analytique nulle d'après le lemme 1.7 .

Proposition 1.11. Si $X$ est un espace $k$-affinoïde irréductible et si $Y$ est un fermé de Zariski irréductible de $X$, alors $\operatorname{dim}_{k} Y+\operatorname{codim}_{\text {Krull }}(Y, X)=\operatorname{dim}_{k} X$; en particulier, $\operatorname{dim}_{k} Y \leqslant \operatorname{dim}_{k} X$ et l'inégalité est stricte si $Y \neq X$.

Démonstration. On procède en deux temps.

1.11.1 Montrons la proposition dans le cas où $\left|k^{*}\right| \neq\{1\}$ et où $X$ est strictement $k$-affinö̈de. Les dimensions en jeu sont alors toutes des dimensions de Krull. Soit $\mathscr{A}$ l'algèbre des fonctions analytique sur $X$; c'est un quotient de $k\left\{T_{1}, \ldots, T_{n}\right\}$ pour un certain $n$. Or tout idéal maximal de $k\left\{T_{1}, \ldots, T_{n}\right\}$ possède un système régulier de paramètres comprenant $n$ éléments [BGR84, $\S 7.1 .1$, Proposition 3]; en conséquence, $k\left\{T_{1}, \ldots, T_{n}\right\}$ est régulière, et tous ses idéaux maximaux ont même hauteur. Elle est donc bi-équidimensionnelle, et il en va de même de son quotient $\mathscr{A}$, ce qui entraîne l'assertion voulue.

1.11.2 Démonstration dans le cas général. On pose $d=\operatorname{dim}_{k} X$. On raisonne par récurrence sur $d$; si $d=0$ la dimension de Krull de $X$ est nulle par le lemme 1.10, et il n'y a rien à démontrer. Supposons le résultat vrai au rang $d-1$. Si $Y=X$ l'assertion souhaitée est triviale; supposons que $Y$ est un fermé strict de $X$ et soit $Y \subset Y_{1} \subset \cdots \subset Y_{l}=X$ une chaîne de fermés de Zariski irréductibles de $X$ telle que $l=\operatorname{codim}_{\text {Krull }}(Y, X)>0$. Soit $f$ une fonction analytique non nulle sur $X$ qui s'annule sur $Y_{l-1}$. Par maximalité de la chaîne $\left(Y_{i}\right)$, le fermé $Y_{l-1}$ est nécessairement une composante irréductible du lieu des zéros de $f$. Soit $\mathbf{r}$ un polyrayon déployant $X$. Le lemme 1.3 assure que $Y_{l-1, \mathbf{r}}$ est une composante irréductible du lieu des zéros de $f$ sur $X_{\mathbf{r}}$. Par le Hauptidealsatz, $\operatorname{codim}_{\text {Krull }}\left(Y_{l-1, \mathbf{r}}, X_{\mathbf{r}}\right)=1$. On a donc $\operatorname{dim}_{k} Y_{l-1}=\operatorname{dim}_{k_{\mathbf{r}}} Y_{l-1, \mathbf{r}}=d-1$, la dernière égalité résultant du 1.11.1 ci-dessus. On peut appliquer l'hypothèse de récurrence à $Y_{l-1}$; il vient

$$
\operatorname{dim}_{k} Y+\operatorname{codim}_{\text {Krull }}\left(Y, Y_{l-1}\right)=d-1 ;
$$

par ailleurs codim $\operatorname{Krull}_{(}\left(Y, Y_{l-1}\right)$ vaut exactement $l-1$ par choix de la suite $\left(Y_{i}\right)$. En conséquence $\operatorname{dim}_{k} Y+l-1=d-1$, d'où l'on conclut que $\operatorname{dim}_{k} Y+l=d$.

Le lemme qui suit est certainement bien connu.

Lemme 1.12. Soit $X$ un espace $k$-affinoïde, soit $V$ un domaine affinoïde de $X$ et soit $\left(X_{1}, \ldots, X_{n}\right)$ la famille des composantes irréductibles de $X$ qui rencontrent $V$. On a alors

$$
\operatorname{dim}_{k} V=\sup _{1 \leqslant i \leqslant n} \operatorname{dim}_{k} X_{i} .
$$




\section{A. Ducros}

Démonstration. Le lemme 1.3 permet de remplacer $k$ par $k_{\mathbf{r}}$ pour un polyrayon $k$-libre $\mathbf{r}$ convenable, et donc de supposer que $\left|k^{*}\right| \neq\{1\}$ et que $X$ et $V$ sont strictement $k$-affinoïdes; toutes les dimensions $k$-analytiques en jeu sont alors des dimensions de Krull. Soit $\mathscr{A}$ (respectivement $\mathscr{B}$ ) l'algèbre des fonctions de $X$ (respectivement $V$ ), soit $\mathscr{X}$ le spectre de $\mathscr{A}$, et pour tout $i$, soit $\mathscr{X}_{i}$ la composante irréductible de $\mathscr{X}$ qui correspond à $X_{i}$. Soit $\mathfrak{m}$ un idéal maximal de $\mathscr{B}$, et soit $x$ le point rigide de $V$ qui lui est associé; désignons par x l'image de $x$ sur $\mathscr{X}$. L'on dispose des isomorphismes suivants

$$
\widehat{\mathscr{B}_{\mathfrak{m}}} \simeq \widehat{\mathscr{O}_{V, x}} \simeq \widehat{\mathscr{O}_{X, x}} \simeq \widehat{\mathscr{O}_{\mathscr{X}, \mathrm{x}}}
$$

le premier et le dernier proviennent de [BGR84, §7.3.2, Proposition 3]; le second se déduit du fait que le point rigide $x$ appartient à l'intérieur topologique de $V$ dans $X$. En conséquence, $\operatorname{dim} \mathscr{B}_{\mathfrak{m}}=\operatorname{dim}_{\mathbf{x}} \mathscr{X}=\sup _{i, \mathbf{x} \in \mathscr{X}_{i}} \operatorname{dim} \mathscr{X}_{i}$.

On en déduit que $\operatorname{dim} \mathscr{B}$ est égal à $\sup _{i \in J} \operatorname{dim} \mathscr{X}_{i}$, où $J$ est l'ensemble des indices $i$ tels que $V \cap X_{i}$ contienne un point rigide. Mais par le Nullstellenstaz analytique, $J=\{1, \ldots, n\}$.

DÉfinition 1.13 (Berkovich). Il découle du lemme précédent que la dimension $k$-analytique d'un espace $k$-affinoïde est supérieure ou égale à celle de chacun de ses domaines affinoïdes. Si $X$ est un espace $k$-analytique quelconque, on appelle dimension $k$-analytique $d e X$, et l'on note $\operatorname{dim}_{k} X$, la borne supérieure des dimensions $k$-analytiques des domaines $k$-affinoïdes de $X$; cette définition est, d'après la remarque qui précède, compatible avec celle déjà donnée pour les espaces $k$-affinoïdes.

1.14 Si $X$ est un espace $k$-analytique, on a $\operatorname{dim}_{k} X=\sup _{x \in X} \mathrm{~d}(\mathscr{H}(x) / k)$ : il suffit en effet de le prouver dans le cas affinoïde, ce qui a été fait par Berkovich [Ber93, lemme 2.5.2]. On en déduit que pour tout recouvrement $\left(X_{i}\right)$ de $X$ par des domaines affinoïdes, $\operatorname{dim}_{k} X=\sup _{\operatorname{dim}_{k}} X_{i}$; ceci entraîne que si $L$ est une extension complète de $k$, alors $\operatorname{dim}_{L} X_{L}=\operatorname{dim}_{k} X$ : le choix d'un recouvrement affinoïde de $X$ permet en effet de ramener le problème au cas (déjà traité, $c f .1 .8$ ) où $X$ est lui-même $k$-affinoïde.

Lemme 1.15. Soit $X$ un espace $k$-affinoïde irréductible et soit $d$ sa dimension $k$-analytique. Si $V$ est un domaine analytique non vide de $X$, alors $\operatorname{dim}_{k} V=d$; si $Y$ est un fermé de Zariski strict de $X$, il ne contient aucun domaine analytique non vide de $X$ et est en particulier d'intérieur vide.

Démonstration. Si $V$ est un domaine analytique de $X$, tout domaine affinö̈de non vide de $V$ est un domaine affinoïde non vide de $X$ et a donc pour dimension $k$-analytique $d$ d'après le lemme 1.12 , d'où la première assertion. Pour la seconde, donnons-nous un domaine analytique non vide $V$ de $X$. Comme $\operatorname{dim}_{k} V=d$ d'après ce qui précède, il existe $x$ dans $V$ tel que $\mathrm{d}(\mathscr{H}(x) / k)=d$; la proposition 1.11 assure en particulier que $\operatorname{dim}_{k} Y<d$, et $x$ n'est donc pas situé sur $Y$.

DÉfinition 1.16 (Berkovich). Soit $X$ un espace $k$-analytique et soit $x$ un point de $X$. On appelle dimension $k$-analytique de $X$ en $x$, et l'on note $\operatorname{dim}_{k, x} X$, le minimum des dimensions $k$-analytiques des domaines affinoïdes de $X$ qui contiennent $x$; il est immédiat que $\operatorname{dim}_{k, x} X \leqslant \operatorname{dim}_{k} X$.

1.17 Si $X$ est affinoïde, on déduit du lemme 1.12 que $\operatorname{dim}_{k, x} X$ est égal au maximum des dimensions $k$-analytiques des composantes irréductibles de $X$ qui contiennent $x$.

Remarque 1.18. Soient $X$ un espace $k$-analytique et $x$ un point de $X$. Soit $L$ une extension complète de $k$ et soit $y \in X_{L}$ situé au-dessus de $x$. Si $W$ est un domaine affinoïde de $X$, alors $\operatorname{dim}_{L} W_{L}=$ $\operatorname{dim}_{k} W$ d'après le 1.8; il en résulte l'inégalité $\operatorname{dim}_{L, y} X_{L} \leqslant \operatorname{dim}_{k, x} X$.

Remarque 1.19. Soit $X$ un espace $k$-affinoïde, soit $Y$ un fermé de Zariski de $X$ et soit $y \in Y$. Du 1.17 ci-dessus et de la proposition 1.11 découle l'inégalité $\operatorname{dim}_{k, y} Y \leqslant \operatorname{dim}_{k, y} X$. 


\section{VARIATION DE LA DIMENSION RELATIVE}

Remarque 1.20. Soit $X$ un espace $k$-analytique, soit $U$ un domaine analytique de $X$ et soit $x$ appartenant à $U$. L'ensemble des domaines affinoïdes de $U$ contenant $X$ est cofinal dans l'ensemble des domaines affinoïdes de $X$ contenant $x$; comme $\operatorname{dim}_{k} V \leqslant \operatorname{dim}_{k} W$ pour tout couple $(V, W)$ de domaines affinoïdes de $X$ avec $V \subset W$, on en déduit que $\operatorname{dim}_{k, x} X=\operatorname{dim}_{k, x} U$.

Lemme 1.21. Soit $X$ un espace $k$-analytique et soit $x$ appartenant à $X$ :

(i) si $\operatorname{dim}_{k, x} X=0$, alors $x$ est rigide et isolé dans $X$;

(ii) si $x$ est isolé dans $X$, alors $\operatorname{dim}_{k, x} X=\mathrm{d}(\mathscr{H}(x) / k)$.

En particulier, $\operatorname{dim}_{k, x} X=0$ si et seulement si $x$ est rigide et isolé dans $X$.

Démonstration. Montrons (i). Supposons que $\operatorname{dim}_{k, x} X=0$. Il existe une famille finie $\left(V_{i}\right)$ de domaines affinoïdes de $X$ contenant $x$ et tels que $\bigcup V_{i}$ soit un voisinage de $x$. Fixons $i$; comme $\operatorname{dim}_{k, x} V_{i}=0$ d'après la remarque 1.20 , les seules composantes irréductibles de $V_{i}$ qui contiennent $x$ sont de dimension $k$-analytique nulle ( $c f .1 .17)$. En vertu du lemme 1.10, une telle composante est constituée d'un nombre fini de points rigides. On en déduit que $x$ est rigide, et isolé dans $V_{i}$; ceci valant pour tout $i$, le point $x$ est isolé dans $X$, et (i) est établi.

Prouvons (ii). On suppose que $x$ est isolé dans $X$. Comme $\{x\}$ est un voisinage ouvert de $x$ dans $X$, il contient un domaine affinoïde non vide de $X$. Autrement dit, $\{x\}$ est un domaine affinoïde de $X$, et $\operatorname{dim}_{k}\{x\}=\mathrm{d}(\mathscr{H}(x) / k)$ par le 1.14. On a alors

$$
\operatorname{dim}_{k, x} X=\operatorname{dim}_{k, x}\{x\}=\operatorname{dim}_{k}\{x\}=\mathrm{d}(\mathscr{H}(x) / k) .
$$

L'assertion ci-dessus est prouvée, d'une manière différente, dans des notes non publiées de Berkovich.

Proposition 1.22. Soit $X$ un espace $k$-analytique, soit $L$ une extension complète de $k$, soit $y$ un point de $X_{L}$ et soit $x$ son image sur $X$. On a l'égalité $\operatorname{dim}_{k, x} X=\operatorname{dim}_{L, y} X_{L}$.

Démonstration. Posons $d=\operatorname{dim}_{k, x} X$. La remarque 1.20 permet de supposer que $X$ est $k$-affinoïde et que $\operatorname{dim}_{k} X=d$. D'après le 1.17, il existe une composante irréductible $Z$ de $X$ de dimension $k$-analytique $d$ qui contient $x$. Soit $\mathbf{r}$ un polyrayon déployant $X$; par le lemme $1.3, Z_{\mathbf{r}}$ est une composante irréductible de $X_{\mathbf{r}}$, et l'on a l'égalité $\operatorname{dim}_{\text {Krull }} Z_{\mathbf{r}}=\operatorname{dim}_{k} Z=d$. Soit $F$ une extension complète de $k$ dans laquelle se plongent $L$ et $k_{\mathbf{r}}$ (on peut par exemple prendre $F$ de la forme $\mathscr{H}(\omega)$, où $\omega$ est un point de l'espace non vide $\mathscr{M}\left(L_{\mathbf{r}}\right)$ ), et soit $t$ un point de $X_{F}$ situé au-dessus de $y$. Soit $T$ une composante irréductible de $\left(Z_{\mathbf{r}}\right)_{F}$ contenant $t$. On a $\operatorname{dim}_{F} T=\operatorname{dim}_{\text {Krull }} T=d$, la première égalité provenant du fait que $T$ est strictement $F$-affinoïde, et la seconde du lemme 2.1.5 de [Con99]. On peut dès lors écrire

$$
d=\operatorname{dim}_{F} X_{F} \geqslant \operatorname{dim}_{t, F} X_{F} \geqslant \operatorname{dim}_{t, F} T=d,
$$

la seconde inégalité provenant de la remarque 1.19 ; en vertu de la remarque 1.18 , on a

$$
d=\operatorname{dim}_{t, F} X_{F} \geqslant \operatorname{dim}_{y, L} X_{L} \geqslant \operatorname{dim}_{k, x} X=d .
$$

Lemme 1.23. Soit $X$ un espace $k$-analytique et soit $x$ un point de $X$. La dimension $d u \mathscr{H}(x)$-espace vectoriel $\Omega_{X / k} \otimes \mathscr{H}(x)$ est supérieure ou égale à $\operatorname{dim}_{k, x} X$.

Démonstration. La proposition ci-dessus permet de vérifier cette affirmation après extension des scalaires et la remarque 1.20 autorise à restreindre $X$. On peut donc supposer que $\left|k^{*}\right| \neq\{1\}$, que $X$ est strictement $k$-affinoïde et que $\mathscr{H}(x)=k$. Si $\mathscr{X}$ désigne le spectre de l'algèbre associé à $X$, et si l'on note $\mathbf{x}$ l'image de $x$ sur $\mathscr{X}$, alors $\operatorname{dim}_{k, x} X=\operatorname{dim}_{\mathbf{x}} \mathscr{X}$ par le lemme 1.12. D'autre part $\widehat{\mathscr{O}_{\mathscr{X}, \mathrm{x}}} \simeq \widehat{\mathscr{O}_{X, x}}(\mathrm{cf}$. [BGR84, $\S 7.3 .2$, proposition 3]); en conséquence,

$$
\operatorname{dim} \mathscr{O}_{X, x}=\operatorname{dim} \mathscr{O}_{\mathscr{X}, \mathbf{x}}=\operatorname{dim}_{k, x} X .
$$




\section{A. Ducros}

Quant à $\Omega_{X / k} \otimes_{x} k$, il s'identifie par le procédé habituel à $\mathfrak{m} / \mathfrak{m}^{2}$, où $\mathfrak{m}$ est l'idéal maximal de $\mathscr{O}_{X, x}$. Le lemme s'ensuit aussitôt.

\section{La dimension relative}

Les résultats établis au paragraphe précédent étant pour la plupart intuitifs, nous nous permettrons, pour alléger la rédaction, de les utiliser systématiquement dans la suite de l'article sans nécessairement donner à chaque fois la référence correspondante.

1.24 Soit $\varphi: Z \rightarrow T$ un morphisme entre deux espaces $k$-analytiques et soit $t$ un point de $T$. La fibre de $\varphi$ en $t$ sera notée ou bien $\varphi^{-1}(t)$, ou bien $Z_{t}$; c'est un espace $\mathscr{H}(t)$-analytique. Soit $z$ appartenant à $\varphi^{-1}(t)$. On désignera par $\operatorname{dim}_{z} \varphi$ la dimension $\mathscr{H}(t)$-analytique de $\varphi^{-1}(t)$ en $z$, que l'on appellera aussi dimension de $\varphi$ en $z$. La fonction $z \mapsto \operatorname{dim}_{z} \varphi$ sera parfois appelée la dimension relative de $\varphi$.

1.25 Soit $Z \rightarrow Y$ un morphisme d'espaces $k$-analytiques dont la dimension relative est identiquement nulle, soit $z$ appartenant à $Z$ et soit $y$ l'image de $z$ sur $Y$. Le corps $\mathscr{H}(z)$ est une extension finie de $\mathscr{H}(y)$; en conséquence $d(\mathscr{H}(z) / \mathscr{H}(y))=0$, et $d(\mathscr{H}(z) / k)=d(\mathscr{H}(y) / k)$; on en déduit l'inégalité $\operatorname{dim}_{k} Z \leqslant \operatorname{dim}_{k} Y$.

Donnons-nous maintenant une flèche $\varphi$ de $Y$ vers un espace $k$-analytique $X$, et notons $\psi$ la composée de $\varphi$ et de $Z \rightarrow Y$. Considérons à nouveau un point $z$ de $Z$, dont on appelle $y$ et $x$ les images respectives sur $Y$ et $X$. Choisissons un domaine affinoïde $V$ de $Y_{x}$ contenant $y$ et tel que

$$
\operatorname{dim}_{\mathscr{H}(x)} V=\operatorname{dim}_{\mathscr{H}(x), y} Y_{x}=\operatorname{dim}_{y} \varphi .
$$

Soit $W$ l'image réciproque de $V$ sur $Z_{x}$; c'est un domaine analytique de $Z_{x}$ qui contient $z$. La dimension relative de $W \rightarrow V$ étant identiquement nulle, $\operatorname{dim}_{\mathscr{H}(x)} W \leqslant \operatorname{dim}_{\mathscr{H}(x)} V$ par ce qui précède. On peut donc écrire

$$
\operatorname{dim}_{z} \psi=\operatorname{dim}_{\mathscr{H}(x), z} Z_{x}=\operatorname{dim}_{\mathscr{H}(x), z} W \leqslant \operatorname{dim}_{\mathscr{H}(x)} W \leqslant \operatorname{dim}_{\mathscr{H}(x)} V=\operatorname{dim}_{y} \varphi .
$$

Lemme 1.26. Soit $\varphi: Y \rightarrow X$ un morphisme entre espaces $k$-affinoïdes non vides et soit $d$ le minimum des $\operatorname{dim}_{y} \varphi$, où y parcourt $Y$. Soit $U$ un ouvert de Zariski de $Y$ tel que $\operatorname{dim}_{y} \varphi=d$ pour tout $y$ appartenant à $U$; on appelle $F$ le fermé complémentaire de $U$ dans $Y$. Pour tout $y$ dans $F$ on a

$$
\operatorname{dim}_{y} \varphi=\max \left(d, \operatorname{dim}_{y} \varphi_{\mid F}\right) .
$$

Démonstration. Soit $y$ appartenant à $F$ et soit $x$ son image sur $X$. Si $W$ est une composante irréductible de $F_{x}$ contenant $y$, elle est incluse dans au moins une composante irréductible de $Y_{x}$

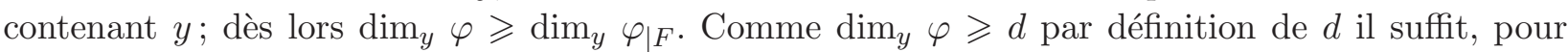
établir le lemme, de montrer que $\operatorname{dim}_{y} \varphi=d$ ou $\operatorname{dim}_{y} \varphi=\operatorname{dim}_{y} \varphi_{\mid F}$. Soit $Z$ une composante irréductible de $Y_{x}$ contenant $y$ et de dimension $\mathscr{H}(x)$-analytique égale à $\operatorname{dim}_{y} \varphi$.

(i) Supposons que $Z \subset F$. Alors $Z$ est contenue dans une composante irréductible de $F_{x}$ contenant $y$, et l'on a donc

$$
\operatorname{dim}_{y} \varphi=\operatorname{dim}_{\mathscr{H}(x)} Z \leqslant \operatorname{dim}_{y} \varphi_{\mid Z} \leqslant \operatorname{dim}_{y} \varphi ;
$$

par conséquent $\operatorname{dim}_{y} \varphi=\operatorname{dim}_{y} \varphi_{\mid Z}$.

(ii) Supposons que $Z$ rencontre $U$. Soit $z$ appartenant à $Z \cap U$. On a alors

$$
d \leqslant \operatorname{dim}_{y} \varphi=\operatorname{dim}_{\mathscr{H}(x)} Z \leqslant \operatorname{dim}_{z} \varphi=d,
$$

cette dernière égalité venant du fait que $z$ appartient à $U$. On en déduit que $\operatorname{dim}_{y} \varphi=d$. 


\section{VARIATION DE LA DIMENSION RELATIVE}

\section{Le Nullstellensatz pour les algèbres affinoïdes}

Lemme 2.1. Soit $\mathscr{A}$ une algèbre $k$-affinoïde et soit $X$ l'espace $\mathscr{M}(\mathscr{A})$. Soit $\left(f_{n}\right)$ une suite de fonctions analytiques inversibles sur $X$ convergeant dans l'algèbre normée $\mathscr{A}$ vers une fonction $f$. Si $X$ est connexe, $f$ est ou bien inversible ou bien nilpotente.

Démonstration. La fonction $f$ est inversible (respectivement nilpotente) si et seulement si $f(x)$ est non nul (respectivement nul) quel que soit $x$ appartenant à $X$; on peut donc raisonner composante par composante et supposer $X$ irréductible. Comme chacune des $f_{n}$ est inversible sa valeur minimale en norme est atteinte sur le bord de Shilov $\operatorname{Sh}(X)$ de $X$ (sur la définition et les propriétés du bord de Shilov, on pourra se reporter à la proposition 2.4.4 de [Ber90]). Si cette valeur est minorée indépendamment de $n$ par un réel strictement positif $R$, alors $|f(x)|$ est supérieure ou égale $R$ pour tout point $x$ de $X$ et $f$ est inversible. Dans le cas contraire, $\operatorname{Sh}(X)$ étant fini, il existe $x \in \operatorname{Sh}(X)$ et une suite extraite $\left(f_{\varphi(n)}\right)$ de $\left(f_{n}\right)$ telle que $f_{\varphi(n)}(x)$ tende vers 0 lorsque $n$ tend vers l'infini. On en déduit que $f(x)=0$.

Soient $x_{1}=x, x_{2}, \ldots, x_{m}$ les points de $\operatorname{Sh}(X)$. Par définition du bord de Shilov, il existe $g$ appartenant à $\mathscr{A}$ telle que $g(x) \neq 0$ et telle que $|g(x)|>\left|g\left(x_{i}\right)\right|$ pour tout $i \geqslant 2$. Soit $V$ le domaine affinoïde de $X$ défini par l'égalité $|g|=|g(x)|$; remarquons que $x \in V$, et donc que $V$ est non vide.

Posons $M=\max _{V}|f|$. Supposons $M$ non nul; il existe alors un entier $N$ tel que l'on ait $M|g(x)|^{N}>\left|f\left(x_{i}\right)\right| \cdot\left|g\left(x_{i}\right)\right|^{N}$ pour tout $i \geqslant 2$. Posons $M^{\prime}=\max _{X}|f| \cdot|g|^{N} ;$ de la majoration évidente $M^{\prime} \geqslant \max _{V}|f| \cdot|g|^{N}=M|g(x)|^{N}$, l'on déduit que $M^{\prime}>0$ et que $M^{\prime}>\left|f\left(x_{i}\right)\right| \cdot\left|g\left(x_{i}\right)\right|^{N}$ pour tout $i \geqslant 2$. Comme le maximum en norme sur $X$ de $f g^{N}$ est atteint $\operatorname{sur} \operatorname{Sh}(X)$, on a nécessairement $\left|f(x) g^{N}(x)\right|=M^{\prime}$, ce qui contredit le fait que $f(x)=0$. Il en découle que $M$ est nul; le lieu des zéros de $f$ est ainsi un fermé de Zariski de l'espace irréductible $X$ qui contient le domaine affinoïde non vide $V$; c'est donc $X$ tout entier, ce qui implique que $f$ est nilpotente.

Corollaire 2.2. Soit $\mathscr{A}$ une algèbre $k$-affinoïde qui est un corps, et soit $X$ l'espace $\mathscr{M}(\mathscr{A})$. Soit $V$ un domaine affinoïde rationnel non vide de $X$, et soit $\mathscr{B}$ l'algèbre des fonctions analytiques sur V. La dimension de Krull de $\mathscr{B}$ est nulle.

Démonstration. Soit $g$ une fonction non nulle de $\mathscr{B}$. Comme $V$ est un domaine rationnel de $X$, on peut écrire $g$ comme une limite de fonctions de $\mathscr{A}$, que l'on peut toujours supposer non nulles et donc inversibles. Par le lemme précédent, la restriction de $g$ à toute composante connexe de $V$ est nilpotente ou inversible, ce qui achève la preuve.

Remarque 2.3. Dans un travail en cours visant à établir les fondements de l'analyse harmonique sur les espaces analytiques de dimension quelconque, Thuillier a démontré, indépendamment de l'auteur, la validité d'un «principe de Harnack» dont le lemme 2.1 ci-dessus est un cas particulier.

\section{Exemples de corps $k$-affinoïdes et Nullstellensatz}

2.4 Soit $\mathbf{r}$ un polyrayon $k$-libre et soit $L$ une extension finie de $k_{\mathbf{r}}$, munie de son unique valeur absolue qui prolonge celle de $k$. L'algèbre $L$ est une $k_{\mathbf{r}}$-algèbre de Banach finie, elle est donc $k$-affinoïde; $\mathscr{M}(L)$ est un singleton $\{x\}$; en termes de semi-norme, $x$ est la valeur absolue de $L$, et $\mathscr{H}(x) \simeq L$. On $\operatorname{aim}_{k} L=\mathrm{d}(L / k)=\mathrm{d}\left(k_{\mathbf{r}} / k\right)=l$, où $l$ est la longueur de $r$.

2.5 On suppose, uniquement dans ce paragraphe, que la valeur absolue de $k$ est triviale. Soient $r$ et $s$ deux réels tels que $0<r \leqslant s<1$; notons $k_{[r ; s]}$ l'algèbre $k$-affinoïde des fonctions analytiques sur la couronne $r \leqslant|T| \leqslant s$. 


\section{A. Ducros}

2.5.1 L'algèbre $k_{[r ; s]}$ est un corps, qui est topologiquement isomorphe à $k((T))$; sa norme envoie une série $f=\sum a_{i} T^{i}$ sur $\max _{a_{i} \neq 0} \max \left(r^{i}, s^{i}\right)=\max \left(r^{v_{T}(f)}, s^{v_{T}(f)}\right)$, où $v_{T}$ est la valuation $T$-adique $\left(v_{T}(0)=-\infty\right.$ par convention). L'espace $\mathscr{M}\left(k_{[r ; s]}\right)$ des valeurs absolues bornées sur $k_{[r ; s]}$ est égal à $\left\{|\cdot|_{t}\right\}_{r \leqslant t \leqslant s}$, où $|\cdot|_{t}$ est la valeur absolue $f \mapsto t^{v_{T}(f)} ;$ l'application $t \mapsto|\cdot|_{t}$ induit un homéomorphisme $[r ; s] \simeq \mathscr{M}\left(k_{[r ; s]}\right)$.

2.5.2 Soit $L$ une extension finie de $k_{[r ; s]}$; on la munit de sa structure de $k_{[r ; s]}$-algèbre de Banach finie, ce qui en fait une algèbre $k$-affinoïde. Soit $x$ appartenant à $\mathscr{M}(L)$. Son image sur $\mathscr{M}\left(k_{[r ; s]}\right)$ est égale à $|\cdot|_{t}$ pour un certain $t \in[r ; s]$; on en déduit que $x$ est nécessairement l'unique valeur absolue $|.|_{t}^{\prime}$ de $L$ qui prolonge $|\cdot|_{t}$. Comme $L$ est une $k_{[r ; s]}$-algèbre de Banach finie, et comme $k_{[r ; s]}$ s'injecte dans $L$, l'application $\mathscr{M}(L) \rightarrow \mathscr{M}\left(k_{[r ; s]}\right]$ est surjective; il s'ensuit que $t \mapsto|\cdot|_{t}^{\prime}$ induit un homéomorphisme $[r ; s] \simeq \mathscr{M}(L)$. Remarquons que pour tout $t \in[r ; s]$, le corps $L$ est déjà complet pour $|.|_{t}^{\prime}$; le corps ultramétrique complet $\mathscr{H}\left(|.|_{t}^{\prime}\right)$ coïncide ainsi avec $\left(L,|.|_{t}^{\prime}\right)$; l'évaluation $L \rightarrow \mathscr{H}\left(|.|_{t}^{\prime}\right)$ est donc un isomorphisme de corps (mais non d'algèbres normées, sauf si $r=s$ ).

L'algèbre $k$-affinoïde $L$ étant réduite, sa norme est équivalente à sa norme spectrale $f \mapsto$ $\max _{r \leqslant t \leqslant s}|f|_{t}^{\prime}=\max \left(|f|_{r}^{\prime},|f|_{s}^{\prime}\right)$. Pour tout $t$, le corps $\mathscr{H}\left(|\cdot|_{t}^{\prime}\right)$ est une extension finie de $\left(k_{[r ; s]},\left.|\cdot|\right|_{t}\right)$, lequel n'est autre que $k_{t}$. En conséquence, $\mathrm{d}\left(\mathscr{H}\left(|\cdot|_{t}^{\prime}\right) / k\right)=1$; ceci valant pour tout $t$, la dimension $k$-analytique de $L$ est égale à 1 .

2.6 On ne fait plus d'hypothèse sur $k$. Une algèbre $k$-affinoïde $L$ sera dite de type I (respectivement de type II) si elle est de la forme décrite au 2.4 (respectivement 2.5.2). Notons qu'une algèbre de type I ou de type II est toujours un corps, qu'il n'existe d'algèbres de type II sur $k$ que si sa valeur absolue est triviale, et que si $\left|k^{*}\right|=\{1\}$ et si $\left.r \in\right] 0 ; 1\left[\right.$, l'algèbre $k_{r}$ est à la fois de type I et II.

ThÉORÈme 2.7 (Nullstellensatz pour les algèbres $k$-affinoïdes). Toute algèbre $k$-affinoïde qui est un corps est de type I ou II.

Démonstration. Soit $\mathscr{A}$ une algèbre $k$-affinoïde qui est un corps et soit $d$ sa dimension $k$-analytique. On raisonne par récurrence sur $d$.

2.7.1 Le cas où d est nulle. L'algèbre $\mathscr{A}$ est alors une $k$-algèbre de Banach finie; comme c'est un corps, c'est une extension finie de $k$ munie de la structure normée donnée par son unique valeur absolue. C'est donc une algèbre de type I.

On suppose $d>0$ et le résultat vrai en dimension analytique égale à $d-1$.

2.7.2 Une première remarque. Si $X$ contient un point rigide $x$, l'évaluation fournit un plongement du corps $\mathscr{A}$ dans $\mathscr{H}(x)$; on en déduit que $\mathscr{A}$ est de dimension finie sur $k$ et donc que $d=0$, ce qui est exclu. Par conséquent, $X$ ne contient aucun point rigide.

2.7.3 Préliminaires. Posons $X=\mathscr{M}(\mathscr{A})$. Par définition d'une algèbre $k$-affinoïde, il existe une immersion fermée $X \hookrightarrow \mathbb{D}$, où $\mathbb{D}$ est un polydisque dont on notera $\left(r_{1}, \ldots, r_{n}\right)$ le polyrayon, et $T_{1}, \ldots, T_{n}$ les fonctions coordonnées. On appelle $J$ l'ensemble des $i$ appartenant à $\{1, \ldots, n\}$ tels que $r_{i}$ soit $k$-libre. Pour tout $i \in\{1, \ldots, n\}$ et tout réel $k$-libre $r$, notons $V_{i, r}$ le domaine rationnel de $X$ défini par l'égalité $\left|T_{i}\right|=r$, et $\mathscr{B}_{i, r}$ son algèbre de fonctions.

Soit $(i, r)$ tel que $V_{i, r}$ soit non vide. D'après le corollaire $2.2, \mathscr{B}_{i, r}$ est de dimension de Krull nulle, et est par ailleurs réduit puisque $X$ est réduit. D'autre part, $V_{i, r}$ est un espace $k_{r}$-affinö̈de. Comme $\mathrm{d}(\mathscr{H}(x) / k)=\mathrm{d}\left(\mathscr{H}(x) / k_{r}\right)+1$ pour tout point $x$ de $V_{i, r}$, l'on a $\operatorname{dim}_{k_{r}} V_{i, r}=\operatorname{dim}_{k} V_{i, r}-1=d-1$, cette dernière égalité venant du fait que $V_{i, r}$ est un domaine affinoïde non vide de l'espace $k$-affinoïde irréductible $X$. L'hypothèse de récurrence, et le fait que $\left|k_{r}^{*}\right| \neq\{1\}$, garantit que $\mathscr{B}_{i, r}$ est un produit 


\section{VARIATION DE LA DIMENSION RELATIVE}

fini d'algèbres $k_{r}$-affinoïdes de type I ; en particulier, l'ensemble sous-jacent à $V_{i, r}$ est fini. Notons une conséquence de ce fait : si $x$ est un point de $V_{i, r}$, c'est un domaine affinoïde de $V_{i, r}$, donc de $X$, et l'on peut ainsi écrire $\mathrm{d}(\mathscr{H}(x) / k)=\operatorname{dim}_{k}\{x\}=d$, et $\mathrm{d}\left(\mathscr{H}(x) / k_{r}\right)=d-1$.

Supposons que $X$ soit la réunion des $V_{i, r}$ où $(i, r)$ parcourt un certain ensemble fini; l'ensemble sous-jacent à $X$ est alors fini. Comme $\mathscr{A}$ est un corps, $X$ est connexe; c'est donc un singleton. Ceci implique qu'il est égal à $V_{i, r}$ pour un certain $(i, r)$, autrement dit que $\mathscr{A}=\mathscr{B}_{i, r}$. Comme $\mathscr{A}$ est un corps, c'est d'après ce qui précède une algèbre $k_{r}$-affinoïde de type I; c'est a fortiori une algèbre $k$-affinoïde de type I.

2.7.4 Preuve lorsque $\left|k^{*}\right| \neq\{1\}$. Soit $V$ le domaine analytique de $X$ défini par les conditions $\left|T_{i}\right|<r_{i}$ pour $i \in J$. C'est un espace strictement $k$-analytique; en vertu de la remarque 2.7 .2 et du Nullstellensatz rigide-analytique, $V$ est vide; cela signifie que $X$ est réunion des $V_{i, r_{i}}$ où $i$ parcourt $J$. D'après le 2.7.3, $\mathscr{A}$ est alors une algèbre $k$-affinoïde de type I.

2.7.5 Preuve lorsque $\left|k^{*}\right|=\{1\}$ et lorsque $d \geqslant 2$. Commençons par une observation : si l'on se donne une famille $\left(\varepsilon_{i}\right)_{1 \leqslant i \leqslant n}$, où $\varepsilon_{i} \in\{0,1\}$ pour tout $i$, l'intersection de $X$ et du lieu de validité sur $\mathbb{D}$ des égalités $\left|T_{i}\right|=\varepsilon_{i}$ est un espace strictement $k$-affinoïde; en vertu de la remarque 2.7 .2 et du Nullstellensatz algébrique (la valeur absolue de $k$ est triviale), il est vide.

Soit $x$ un point de $X$. Par ce qui précède, il existe un réel $r>0$ et différent de 1 (autrement dit, $r$ est $k$-libre) et un entier $i$ tel que $x \in V_{i, r}$. On a vu au 2.7.3 que si $y$ est un point de $V_{i, r}$, alors $\mathrm{d}\left(\mathscr{H}(y) / k_{r}\right)=d-1$. Or par hypothèse, $d-1 \geqslant 1$; dès lors, $V_{i, r}$ ne contient aucun point $k_{r}$-rigide. Soit $J_{r}$ l'ensemble des $j \in\{1, \ldots, n\}$ qui sont $k_{r}$-libres; notons que $J_{r} \subset J$. Le domaine analytique $W$ de $V_{i, r}$ défini par les conditions $\left|T_{j}\right|<r_{j}$ pour $j$ appartenant à $J_{r}$ est strictement $k_{r}$-analytique. Ne contenant aucun point $k_{r}$-rigide, il est vide en raison du Nullstellensatz rigide-analytique. En particulier, $x \notin W$ et il existe donc $j$ dans $J_{r}$ tel que $x$ appartienne à $V_{j, r_{j}}$.

Ainsi, $X$ est réunion des $V_{j, r_{j}}$ où $j$ parcourt $J$. Le 2.7.3 assure alors que $\mathscr{A}$ est une algèbre $k$-affinoïde de type I.

2.7.6 Preuve lorsque $\left|k^{*}\right|=\{1\}$ et lorsque $d=1$. Si l'ensemble des $i \in\{1, \ldots, n\}$ tels que $r_{i}<1$ et $T_{i \mid X} \neq 0$ était vide, le corps $\mathscr{A}$ serait un quotient d'une $k$-algèbre de type fini, et serait donc une extension finie de $k$; on aurait ainsi $\operatorname{dim}_{k} \mathscr{A}=0$, ce qui est exclu. L'une des fonctions coordonnées, que l'on va noter $f$ pour simplifier, induit ainsi une fonction non nulle, donc inversible, sur $X$, dont la norme spectrale est strictement inférieure à 1 .

La fonction $f$ définit un morphisme $X \rightarrow \mathbb{A}_{k}^{1, a n}$, dont l'image est inclus dans l'ouvert $0<|T|<1$ de $\mathbb{A}_{k}^{1, a n}$, que l'on peut également décrire comme $\left\{|\cdot|_{t}\right\}_{0<t<1}$, où $|\cdot|_{t}=f \mapsto t^{v_{T}(f)}$. L'espace $X$ étant connexe, compact, et non vide, $f(X)$ est de la forme $\left\{|\cdot|_{t}\right\}_{r \leqslant t \leqslant s}$ pour $r$ et $s$ convenables, vérifiant $0<r \leqslant s<1$. Autrement dit, $f$ induit un morphisme surjectif $p: X \rightarrow \mathscr{M}\left(k_{[r ; s]}\right)$, qui permet entre autres de voir $\mathscr{A}$ comme une $k_{[r ; s]}$-algèbre.

Fixons $t \in[r ; s]$. Pour tout $x$ appartenant à $p^{-1}\left(|\cdot| t_{t}\right)$, l'on a

$$
\operatorname{dim}_{k} X=1 \geqslant \mathrm{~d}(\mathscr{H}(x) / k)=\mathrm{d}\left(\mathscr{H}(x) / \mathscr{H}\left(|\cdot|_{t}\right)\right)+1,
$$

et donc $\mathrm{d}\left(\mathscr{H}(x) / \mathscr{H}\left(|\cdot|_{t}\right)\right)=0$. La dimension $\mathscr{H}\left(|\cdot|_{t}\right)$-analytique de $p^{-1}\left(|\cdot|_{t}\right)$ est de ce fait nulle; ce dernier est en conséquence constitué d'un nombre fini de points $\mathscr{H}\left(|\cdot|_{t}\right)$-rigides. Soit $y$ l'un de ces points $\left(p^{-1}\left(|\cdot|_{t}\right)\right.$ est non vide par choix de $\left.t\right)$. L'évaluation définit un $k_{[r ; s]}$ plongement de $\mathscr{A}$ dans $\mathscr{H}(y)$. Comme $k_{[r ; s]} \rightarrow \mathscr{H}\left(|\cdot|_{t}\right)$ est un isomorphisme de corps, $\mathscr{A}$ est finie sur $k_{[r ; s]}$.

Pour tout $t \in[r ; s]$, la fibre $p^{-1}\left(|\cdot|_{t}\right)$ comprend au plus un élément, à savoir l'unique prolongement $|\cdot|_{t}^{\prime}$ de $|\cdot|_{t}$ à $\mathscr{A}$, et au moins un, puisque $p$ est surjective. On en déduit que $\mathscr{M}(\mathscr{A})$ est exactement $\left\{|\cdot|_{t}^{\prime}\right\}_{t \in[r ; s]}$. L'algèbre $k$-affinoïde $\mathscr{A}$ étant réduite, sa structure d'algèbre normée peut être définie 


\section{A. Ducros}

par sa norme spectrale, qui envoie $f$ sur $\max _{r \leqslant t \leqslant s}|f|_{t}^{\prime}=\max \left(|f|_{r}^{\prime},|f|_{s}^{\prime}\right)$. L'algèbre $k$-affinoïde $\mathscr{A}$ est donc de type II.

\section{Une version analytique du Main Theorem de Zariski}

\section{La réduction des germes ponctuels d'espaces analytiques}

C'est une théorie développée par Temkin [Tem04] que nous utiliserons librement. Si $K$ est une extension valuée de $k$, on notera $\widetilde{K}^{\bullet}$ le corps résiduel gradué associée, et $\mathbb{P}_{\widetilde{K}} \bullet$ l'ensemble des valuations graduées de $\widetilde{K}^{\bullet}$ dont l'anneau contient $\widetilde{k}^{\bullet}$. Si F est un ensemble d'éléments homogènes de $\widetilde{K}^{\bullet}$, on désignera par $\mathbb{P}_{\widetilde{K}} \bullet\{F\}$ la partie de $\mathbb{P}_{\widetilde{K}} \bullet$ formée des valuations dont l'anneau contient $F$. On munit $\mathbb{P}_{\widetilde{K}} \bullet$ de la topologie engendrée par les $\mathbb{P}_{\widetilde{K}} \bullet\{F\}$ avec $\mathrm{F}$ fini; un ouvert de $\mathbb{P}_{\widetilde{K}}$. sera qualifié d' affine s'il est égal à $\mathbb{P}_{\widetilde{K}} \bullet\{F\}$ pour un certain $F$ fini.

À tout germe ponctuel $(X, x)$ d'espace $k$-analytique, Temkin associe fonctoriellement un espace topologique connexe, non vide et quasi-compact $\widetilde{(X, x)}$ muni d'une application continue vers $\mathbb{P} \widetilde{\mathscr{H}(x)}$. qui est un homéomorphisme local, et une immersion ouverte lorsque $(X, x)$ est séparé (cf. [Tem04, Proposition 4.8(iii)]).

Lemme 3.1. Soit $K \hookrightarrow L$ un morphisme fini entre extensions valuées de $k$; si l'extension finie $\widetilde{K} \hookrightarrow \widetilde{L}$ est purement inséparable, $\mathbb{P}_{\widetilde{L} \bullet} \rightarrow \mathbb{P}_{\widetilde{K}} \bullet$ est un homéomorphisme qui transforme tout ouvert affine en un ouvert affine.

\section{Démonstration.}

3.1.1 Soit $\varpi$ un élément homogène de $\widetilde{L}^{\bullet}$. C'est l'image d'un certain $\lambda$ appartenant à $L$; le degré de $\varpi$ est alors égal à $|\lambda|$. Comme $L / K$ est finie, il existe un entier $n$ et un élément $\kappa$ de $K$ tels que $\left|\lambda^{n} / \kappa\right|=1$. Le corps $\widetilde{L}$ étant purement inséparable sur $\widetilde{K}$, il existe un entier $m$ (puissance de l'exposant caractéristique de $\widetilde{k})$, et un élément $\mu$ de $K$, tels que $|\mu|=1$ et ${\widetilde{\left(\lambda^{n} / \kappa\right)}}^{m}=\widetilde{\mu}$. On en déduit que $\varpi^{n m}$ est l'élément homogène de degré $|\lambda|^{n m}$ de $\widetilde{K}^{\bullet}$ défini comme l'image de $\kappa^{m} \mu$.

3.1.2 La flèche $\mathbb{P}_{\widetilde{L}} \bullet \rightarrow \mathbb{P}_{\widetilde{K}}$. est injective. Soient $\mathscr{O}$ et $\mathscr{O}^{\prime}$ deux anneaux de valuation de $\widetilde{L}^{\bullet}$ contenant $\widetilde{k}^{\bullet}$ et dont les intersections avec $\widetilde{K}^{\bullet}$ coïncident. Soit $\varpi$ un élément homogène de $\widetilde{L}^{\bullet}$. D'après le 3.1 .1 , il existe un entier non nul $s$ tel que $\varpi^{s}$ soit un élément (homogène) de $\widetilde{K}^{\bullet}$. Or $\varpi$ appartient à $\mathscr{O}\left(\right.$ respectivement $\left.\mathscr{O}^{\prime}\right)$ si et seulement si $\varpi^{s}$ appartient à $\mathscr{O} \cap \widetilde{K}^{\bullet}$ (respectivement $\mathscr{O}^{\prime} \cap \widetilde{K}^{\bullet}$ ). On en déduit que $\mathscr{O}$ et $\mathscr{O}^{\prime}$ ont les mêmes éléments homogènes, donc qu'ils sont égaux.

3.1.3 La flèche $\mathbb{P}_{\widetilde{L}} \bullet \rightarrow \mathbb{P}_{\widetilde{K}} \bullet$ est surjective. Cela découle de la remarque qui précède le lemme 1.4 de [Tem04].

3.1.4 La flèche $\mathbb{P}_{\widetilde{L}}^{\bullet} \rightarrow \mathbb{P}_{\widetilde{K}} \bullet$ transforme tout ouvert affine en un ouvert affine. Soit $F$ un ensemble fini d'éléments homogènes de $\widetilde{L}^{\bullet}$. Le 3.1.1 assure qu'il existe un entier non nul $s$ tel que $\mathrm{F}^{s}$ (c'est-à-dire l'image de $\mathrm{F}$ sous l'élévation à la puissance $s$ ) soit inclus dans $\widetilde{K}^{\bullet}$. Comme $\mathbb{P}_{\widetilde{L}} \cdot\{\mathrm{F}\}$ est égal à $\mathbb{P}_{\widetilde{L}} \cdot\left\{\mathrm{F}^{s}\right\}$, son image sur $\mathbb{P}_{\widetilde{K}} \bullet$ est simplement $\mathbb{P}_{\widetilde{K}} \bullet\left\{\mathrm{F}^{s}\right\}$, qui est un ouvert affine.

La démonstration du lemme est terminée.

\section{Le Main Theorem}

ThÉorème 3.2. Soit $\varphi: Y \rightarrow X$ un morphisme d'espaces $k$-analytiques; on suppose que $Y$ est séparé. Soit y un point de $Y$. Les propositions suivantes sont équivalentes: 


\section{VARIATION DE LA DIMENSION RELATIVE}

(i) $\operatorname{dim}_{y} \varphi=0$;

(ii) il existe un voisinage $k$-analytique $V$ de $y$ tel que la restriction de $\varphi$ à $V$ admette une factorisation

$$
V \rightarrow W \rightarrow T \rightarrow X
$$

où $V \rightarrow W$ est fini, où $W$ est séparé, où $W \rightarrow T$ identifie $W$ à un domaine analytique de $T$, et où $T \rightarrow X$ est étale.

Si de plus $Y$ est bon, alors dans la proposition (ii) on peut prendre $V$ et $W$ affinoïdes.

Démonstration. L'implication (ii) $\Rightarrow$ (i) est évidente. On va supposer que (i) est vraie, et établir (ii). Notons $x$ l'image de $y$. Comme $\operatorname{dim}_{y} \varphi=0$, le corps $\mathscr{H}(y)$ est une extension finie de $\mathscr{H}(x)$. Soit $l$ la fermeture séparable de $\widetilde{\mathscr{H}(x)}$ dans $\widetilde{\mathscr{H}(y)}$, et soit $L$ l'extension non ramifiée de $\mathscr{H}(x)$ de corps résiduel $l$. Les catégories $\mathscr{H}(x)_{\text {ét }}$ et $(X, x)_{\text {ét }}$ étant équivalentes [Ber93, théorème 3.4.1], il existe un morphisme étale $T \rightarrow X$ dont la fibre en $x$ s'identifie à $\mathscr{M}(L)$. La fibre en $y$ de la flèche étale $T \times_{X} Y \rightarrow Y$ a un point $\mathscr{H}(y)$-rationnel; en conséquence $T \times_{X} Y \rightarrow Y$ possède une section au voisinage de $y$, et l'on peut donc supposer, quitte à restreindre $Y$, que $Y \rightarrow X$ se factorise par $T$. En remplaçant $X$ par $T$, on se ramène au cas $\widetilde{\mathscr{H}(y)}$ est une extension finie purement inséparable de $\widetilde{\mathscr{H}(x)}$.

Dans le diagramme commutatif

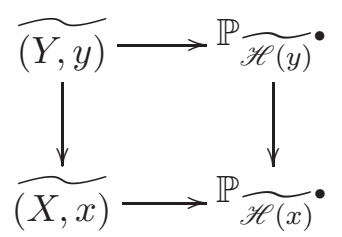

les deux flèches horizontales sont des homéomorphismes locaux, et celle du haut est même une immersion ouverte puisque $(Y, y)$ est séparé ; quant à la flèche verticale de droite, c'est un homéomorphisme en vertu du lemme 3.1.

On en déduit que $\widetilde{(Y, y)} \rightarrow \widetilde{(X, x)}$ est une immersion ouverte; dès lors, on peut par le théorème 4.5 de [Tem04] l'identifier à $\widetilde{(Z, x)} \rightarrow \widetilde{(X, x)}$ pour un certain domaine analytique séparé $(Z, x)$ de $(X, x)$. La flèche $(Y, y) \rightarrow(X, x)$ se factorise par $(Z, x)$ d'après la proposition 4.6 de [Tem04]. Ceci permet de remplacer $(X, x)$ par $(Z, x)$, et de supposer à partir de maintenant que le diagramme ci-dessus est cartésien.

On déduit dès lors du théorème 5.2 de [Tem04] que le point y appartient à l'intérieur du morphisme $\varphi$; celui-ci est donc fini en $y$ (cf. [Ber93, Proposition 3.1.4]), ce qui achève de prouver (ii). Si $Y$ est bon, alors $\widetilde{(Y, y)}$ s'identifie à un ouvert affine de $\mathbb{P}_{\mathscr{H}(y)}$ d'après le théorème 5.1 de [Tem04]. En conséquence, $\widetilde{(X, x)}$ s'identifie par le Lemme 3.1 à un ouvert affine de $\mathbb{P}_{\mathscr{H}(x)}$, et l'on utilise à nouveau le théorème 5.1 de [Tem04] pour conclure que $(X, x)$ est bon, ce qui établit la dernière assertion du lemme.

\section{Le lieu d'annulation de la dimension relative}

3.3 On dira qu'un morphisme $\mathrm{A} \rightarrow \mathrm{B}$ de $k$-algèbres est élémentairement radiciel si $\mathrm{B}$ est $\mathrm{A}$ isomorphe à $\mathrm{A}[T] /\left(T^{p^{n}}-a\right)$ pour un certain entier $n$ et un certain $a$ dans $A$; notons que si $p=1$, cela signifie simplement que $A \rightarrow B$ est un isomorphisme. On dira qu'un morphisme de $A \rightarrow B$ de $k$-algèbres se dévisse bien s'il admet une factorisation de la forme $A \rightarrow A / I \rightarrow A^{\sharp} \rightarrow B$, où I est un idéal de $A$, où $A / I \rightarrow A^{\sharp}$ est fini étale, et où $A^{\sharp} \rightarrow B$ est composé de flèches élémentairement radicielles. 


\section{A. Ducros}

Remarquons que dans cette situation $A^{\sharp}$ s'injecte dans $B$; en conséquence, si $B$ est intègre, $A^{\sharp}$ l'est aussi, et l'on peut alors supposer I premier et $A / I \rightarrow A^{\sharp}$ injective.

On dira qu'un morphisme fini entre $k$-schémas affines (respectivement espaces $k$-affinoïdes) se dévisse bien si c'est le cas du morphisme de $k$-algèbres correspondant.

Lemme 3.4. Soit $Y \rightarrow X$ un morphisme fini d'espaces $k$-affinoïdes; on note $\mathscr{X}$ et $\mathscr{Y}$ les spectres respectifs des $k$-algèbres associées à $X$ et $Y$, et $\rho_{X}$ (respectivement $\rho_{Y}$ ) l'application continue $X \rightarrow \mathscr{X}$ (respectivement $Y \rightarrow \mathscr{Y}$ ). Soit y un point de $Y$ tel que $\mathscr{O}_{Y, y}$ soit réduit, et tel que $\rho_{Y}(y)$ soit le point générique d'une composante irréductible de $\mathscr{Y}$; soit $x$ l'image de y sur $X$. Il existe un voisinage affinoïde $X^{\prime}$ de $x$ dans $X$, et un voisinage affinö̈de intègre $Y^{\prime}$ de $y$ dans $Y \times_{X} X^{\prime}$, tels que $Y^{\prime} \rightarrow X^{\prime}$ soit fini et se dévisse bien.

Démonstration. Quitte à remplacer $Y$ par l'unique composante irréductible qui contient $y$, on peut supposer que $\mathscr{Y}$ est irréductible; dans ce cas, $\rho_{Y}(y)$ est son point générique. Comme $\mathscr{O}_{Y, y}$ est réduit, le schéma $\mathscr{Y}$ est réduit au voisinage de $\rho_{Y}(y)$ en vertu du théorème 2.2.1 de [Ber93]. On en déduit que $\mathscr{Y} \rightarrow \mathscr{X}$ se factorise par un sous-schéma fermé de $\mathscr{X}$ de support $\overline{\left\{\rho_{X}(x)\right\}}$ qui est réduit au voisinage de $\rho_{X}(x)$. La flèche $\kappa\left(\rho_{X}(x)\right) \hookrightarrow \kappa\left(\rho_{Y}(y)\right)$ est une extension finie de corps, et en conséquence se dévisse bien. On en déduit, par les raisonnements usuels relatifs aux limites projective de schémas, l'existence d'un voisinage affine $\mathscr{U}$ de $\rho_{X}(x)$ dans $\mathscr{X}$, tel que $\mathscr{Y} \times \mathscr{X} \mathscr{U} \rightarrow \mathscr{U}$ se dévisse bien.

D'après le théorème 2.2.1 de [Ber93], le lieu régulier Reg $Y$ de l'espace annelé $Y$ en est un ouvert de Zariski qui contient $\rho^{-1}\left(\rho_{Y}(y)\right)$, et en particulier tous les antécédents de $x$. L'image de $Y-\operatorname{Reg} Y$ sur $X$ est donc un compact qui évite $x$; en conséquence, il existe un voisinage affinoïde $X^{\prime}$ de $x$ dans $X$, inclus dans $\rho_{X}^{-1}(\mathscr{U})$, et dont l'image réciproque sur $Y$ est contenue dans $\operatorname{Reg} Y$. Le domaine affinoïde $Y \times_{X} X^{\prime}$ de $Y$ est un espace annelé régulier par le corollaire 2.2.8 de [Ber93], et par construction $Y \times_{X} X^{\prime} \rightarrow X^{\prime}$ est fini et se dévisse bien. Si $Y^{\prime}$ désigne la composante connexe de $y$ dans $Y \times_{X} X^{\prime}$, alors $Y^{\prime} \rightarrow X^{\prime}$ est fini et se dévisse bien; comme $Y^{\prime}$ est régulier en tant qu'espace localement annelé, il est intègre (on utilise encore le théorème 2.2.1 de [Ber93]).

Proposition 3.5. Soit $\varphi: Y \rightarrow X$ un morphisme d'espaces $k$-analytiques. L'ensemble $\mathrm{E}$ des points $y$ de $Y$ en lesquels $\varphi$ est de dimension nulle est un ouvert de Zariski de $Y$.

Démonstration. La question est locale pour la G-topologie, ce qui permet de se ramener au cas où $X$ et $Y$ sont affinoïdes; la dimension étant insensible aux phénomènes de nilpotence, on peut faire l'hypothèse que $Y$ est réduit. On raisonne alors par récurrence noethérienne, ce qui permet de supposer que la proposition est vraie pour tous les fermés de Zariski stricts de $Y$. Si E est vide, il n'y a rien à faire; on se place dans le cas où il ne l'est pas, et l'on choisit e $\in \mathrm{E}$.

3.5.5 Construction d'un domaine affinoïde $Y_{1}$ de $Y$ et d'une factorisation agréable de $Y_{1} \rightarrow X$. D'après le théorème 3.2 , il existe un voisinage $k$-affinoïde $Y_{0}$ de e dans $Y$, un espace $k$-analytique $T$, une flèche étale $T \rightarrow X$, et un domaine affinoïde $W$ de $T$ tels que $\varphi$ se factorise par un morphisme fini $Y_{0} \rightarrow W$. Soit $y$ un point de $Y_{0}$ qui n'est situé sur aucun fermé de Zariski strict de sa composante irréductible; comme $Y$ est réduit, $\mathscr{O}_{Y_{0}, y}$ est réduit.

On est dans les conditions d'application du lemme 3.4. Il existe de ce fait un voisinage affinoïde intègre $Y_{1}$ de $y$ dans $Y_{0}$, et un domaine affinoïde $W_{1}$ de $W$, tels que $Y_{0} \rightarrow W$ induise une flèche $Y_{1} \rightarrow W_{1}$ qui est finie et se dévisse bien.

Si $\mathscr{C}$ et $\mathscr{D}$ désignent les algèbres respectivement associées à $W_{1}$ et $Y_{1}$, alors $\mathscr{C} \rightarrow \mathscr{D}$ possède une factorisation de la forme $\mathscr{C} \rightarrow \mathscr{C} / \mathscr{P} \hookrightarrow \mathscr{C}^{\sharp} \hookrightarrow \mathscr{D}$, où $\mathscr{P}$ est un idéal premier de $\mathscr{C}$, où $\mathscr{C} / \mathscr{P} \hookrightarrow \mathscr{C}^{\sharp}$ est finie étale, et où $\mathscr{C}^{\sharp} \hookrightarrow \mathscr{D}$ est composée de morphismes élémentairement radiciels. On en déduit l'existence d'un entier $n$ tel que $\mathscr{D}^{p^{n}}$ soit inclus dans l'image de $\mathscr{C}^{\sharp}$. 


\section{VARIATION DE LA DIMENSION RELATIVE}

3.5.6 Construction d'une factorisation $Y \rightarrow Y^{b} \rightarrow X$, où $Y \rightarrow Y^{b}$ est radiciel. Notons $\mathscr{B}^{b}$ le complété de la sous- $\mathscr{A}$-algèbre de $\mathscr{B}$ engendrée par $\mathscr{B}^{p^{n}}$. Soit $\mathbf{r}$ un polyrayon déployant $\mathscr{B}$. L'algèbre $\mathscr{B}_{\mathbf{r}}^{b}$ est une sous-algèbre de Banach de $\mathscr{B}_{\mathbf{r}}$. Soit $b$ appartenant à $\mathscr{B}_{\mathbf{r}}$ et de norme spectrale inférieure ou égale à 1 . Par définition de $\mathscr{B}^{b}$, l'élément $b^{p^{n}}$ appartient à $\mathscr{B}_{\mathbf{r}}^{b}$; comme cette dernière est une sousalgèbre normée de $\mathscr{B}_{\mathbf{r}}$, la norme spectrale de $b^{p^{n}}$ vu comme appartenant à $\mathscr{B}_{\mathbf{r}}^{b}$ est égale à la norme spectrale de $b^{p^{n}}$ vu comme appartenant à $\mathscr{B}_{\mathbf{r}}$, et est donc majorée par 1 . On en déduit que $\mathscr{B}_{\mathbf{r}}^{b}$ est strictement $k_{\mathbf{r}}$-affinoïde [BGR84, 6.3.3, Proposition 1]; par le corollaire 2.2.8 de [Ber90], l'algèbre $\mathscr{B}^{b}$ est $k$-affinoïde, et son plongement dans $\mathscr{B}$ est par construction fini, radiciel et admissible. Comme

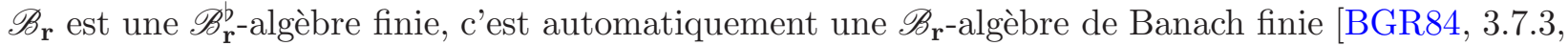
Proposition 3], et $\mathscr{B}$ est donc une $\mathscr{B}^{\text {b}}$-algèbre de Banach finie [Ber90, Proposition 2.1.11].

Soit $\left(f_{1}, \ldots, f_{r}\right)$ un système de générateurs topologiques de la $\mathscr{A}$-algèbre $\mathscr{B}$; pour tout $i$, on note $\xi_{i}$ l'élément $f_{i}^{p^{n}}$ de $\mathscr{B}^{b}$; les $\xi_{i}$ constituent un système de générateurs topologiques de la $\mathscr{A}$-algèbre $\mathscr{B}^{b}$, et les $\mathrm{d} \xi_{i}$ engendrent donc le $\mathscr{B}^{b}$-module $\Omega_{\mathscr{B}^{b}} / \mathscr{A}$. Soit $Y^{b}$ l'espace $\mathscr{M}\left(\mathscr{B}^{b}\right)$. Le morphisme $\varphi$ admet une factorisation $Y \rightarrow Y^{b} \rightarrow X$; la flèche de gauche est finie, radicielle et surjective (puisque $\mathscr{B}^{b}$ s'injecte dans $\mathscr{B}$ ); elle induit un homéomorphisme entre les espaces sous-jacents aussi bien pour leurs topologies naturelles que pour celles de Zariski.

3.5.7 Trivialité de $\Omega_{Y^{b} / X}$ au-dessus d'un ouvert de Zariski non vide de $Y^{b}$. Soit $Y_{1}^{b}$ l'image de $Y_{1}$ dans $Y^{b}$. En écrivant $Y_{1}$ comme une réunion finie de domaines rationnels [Duc03, lemme 2.4], puis en élevant les fonctions en jeu à la puissance $p^{n}$, on voit que $Y_{1}^{b}$ est une réunion finie de domaines rationnels de $Y^{b}$; c'en est en particulier un domaine $k$-analytique, dont l'algèbre des fonctions sera notée $\mathscr{D}^{b}$.

Le morphisme $T \times_{X} Y_{1}^{b} \rightarrow Y_{1}^{b}$ est un revêtement fini étale qui, après le changement de base $Y_{1} \rightarrow Y_{1}^{b}$, admet par construction une section; en vertu de «l'invariance topologique du site étale » [Ber93, proposition 4.3.4], $T \times_{X} Y_{1}^{b} \rightarrow Y_{1}^{b}$ admet déjà une section, ce qui signifie que $Y_{1} \rightarrow T$ se factorise par $Y_{1}^{b}$, et il en va de même de $Y_{1} \rightarrow W_{1}$, puisque $W_{1}$ est un domaine affinoïde de $T$.

Comme $Y_{1}^{b}$ est un domaine analytique de l'espace réduit $Y^{b}$, la surjectivité de $Y_{1} \rightarrow Y_{1}^{b}$ entraîne l'injectivité de $\mathscr{D}^{b} \rightarrow \mathscr{D}$, et $Y_{1} \rightarrow Y_{1}^{b} \rightarrow W_{1}$ induit ainsi un diagramme $\mathscr{C} / \mathscr{P} \hookrightarrow \mathscr{D}^{\mathrm{b}} \hookrightarrow \mathscr{D}$ qui met en jeu trois anneaux intègres. Notons $\mathscr{K}$ le corps des fractions de $\mathscr{C} / \mathscr{P}$.

Fixons $i$. L'image dans $\mathscr{D}$ de l'élément $\xi_{i \mid Y_{1}^{b}}$ de $\mathscr{D}^{b}$ est égale à $f_{i \mid Y_{1}}^{p^{n}}$; elle provient donc de $\mathscr{C}^{\sharp}$, qui est finie étale sur $\mathscr{C} / \mathscr{P}$; il existe par conséquent un polynôme séparable $P_{i}$ à coefficients dans $\mathscr{K}$ tel que $P_{i}\left(f_{i \mid Y_{1}}^{p^{n}}\right)=0$ dans $\mathscr{D} \otimes_{\mathscr{C}} \mathscr{K}$. De ce qui précède, on déduit que l'élément $P_{i}\left(\xi_{i \mid Y_{1}^{\mathrm{b}}}\right)$ de $\mathscr{D}^{\mathrm{b}} \otimes_{\mathscr{C}} \mathscr{K}$ est déjà nul.

Il existe un ouvert de Zariski non vide $U$ de $Y_{1}^{b}$ sur lequel chacun des $P_{i}$ est à coefficients holomorphes et à discriminant inversible. Par construction, $\Omega_{U / W_{1}}$ est nul. Comme $W_{1} \rightarrow T$ est une immersion de domaine affinoïde et comme $T \rightarrow X$ est étale, $\Omega_{U / X}$ est lui aussi trivial.

Soit $Z^{b}$ l'ensemble des points $y$ de $Y^{b}$ en lesquels le rang de $\Omega_{Y^{b} / X}$ est nul. C'est un ouvert de Zariski par le lemme de Nakayama. Comme $Z^{b}$ contient $U$, il est non vide.

3.5.8 Conclusion. La dimension de $Y^{b} \rightarrow X$ est nulle en tout point de $Z^{b}$ par la définition de ce dernier. Soit $Z$ l'image réciproque de $Z^{b}$ sur $Y$; c'est un ouvert de Zariski non vide de $Y$, et comme $Y \rightarrow Y^{b}$ est fini, $\operatorname{dim}_{z} \varphi=0$ pour tout point $z$ de $Z$ d'après le 1.25 . Soit $F$ l'ensemble des points de $Y-Z$ en lesquels la dimension de $\varphi_{\mid Y-Z}$ est strictement positive. Comme $Y-Z$ est un fermé de Zariski strict de $Y$, il satisfait la proposition, et $F$ est ainsi un fermé de Zariski de $Y-Z$, et donc de $Y$. Le lemme 1.26 assure par ailleurs que $F$ est l'ensemble des points de $Y$ en lesquels la dimension de $\varphi$ est strictement positive, ce qui achève la démonstration. 


\section{A. Ducros}

\section{Factorisation à la « Raynaud et Gruson» et semi-continuité de la dimension}

\section{Systèmes de paramètres analytiques}

DÉfinition 4.1. Soit $\mathscr{A}$ une algèbre $k$-affinoïde et soit $x$ un point de $\mathscr{M}(\mathscr{A})$ situé au-dessus d'un point fermé $\mathrm{x}$ de Spec $\mathscr{A}$; posons $n=\operatorname{dim}_{k, x} \mathscr{M}(\mathscr{A})$. On appellera système de paramètres $k$-analytiques en $x$ tout $n$-uplet $\left(h_{1}, \ldots, h_{n}\right)$ de fonctions de $\mathscr{A}$ telles que la dimension en $x$ du morphisme $\mathscr{M}(\mathscr{A}) \rightarrow \mathbb{A}_{k}^{n}$ défini par les $h_{i}$ soit nulle.

Remarque 4.2. La notion de système de paramètres $k$-analytiques est relative à un espace $k$-affinoïde ambiant, mais ce dernier ne sera explicitement mentionné que si le contexte est ambigü.

Exemple 4.3. On va voir que de tels systèmes existent toujours. Gardons les notations $\mathscr{A}, \mathbf{x}, x$ et $n$ introduites ci-dessus. Notons $\delta$ la dimension de l'anneau local $\mathscr{O}_{\text {Spec } \mathscr{A}, \mathbf{x}}$ (rappelons que $\mathscr{A}$ est noethérien d'après la proposition 2.1.3 de [Ber90]) et choisissons une famille $\left(f_{1}, \ldots, f_{\delta}\right)$ d'éléments de $\mathscr{A}$ tels que $\mathbf{x}$ soit une composante irréductible du lieu des zéros (ensembliste) des $f_{i}$ sur Spec $\mathscr{A}$.

Le point $\mathrm{x}$ correspond à un idéal maximal $\mathfrak{m}$ de $\mathscr{A}$. Le corps quotient $\mathscr{A} / \mathfrak{m}$, que l'on notera $\mathscr{B}$, hérite d'une structure d'algèbre $k$-affinoïde. L'espace $\mathscr{M}(\mathscr{B})$ s'identifie à un fermé de Zariski de $\mathscr{M}(\mathscr{A})$, et si $l$ désigne la dimension $k$-analytique de $\mathscr{M}(\mathscr{B})$ alors $l+\delta=n$; par hypothèse, $\mathscr{M}(\mathscr{B})$ contient $x$. Soit $\varphi$ la flèche Spec $\mathscr{A} \rightarrow \mathbb{A}_{k}^{\delta}$ induite par les $f_{i}$; par choix de ces derniers, le point $\mathbf{x}$ est isolé dans $\varphi^{-1}(O)$, où $O$ est l'origine. En conséquence, $\mathscr{M}(\mathscr{B})$ est topologiquement (c'est-à-dire sans tenir compte des multiplicités) une composante connexe de $\left(\varphi^{a n}\right)^{-1}(O)$.

Puisque $\mathscr{B}$ est à la fois une algèbre $k$-affinoïde et un corps, le théorème 2.7 assure qu'elle est de type I ou II; ces deux types sont respectivement décrits au 2.4 et au 2.5.2; on utilise ci-dessous sans rappels ni justification l'ensemble des résultats établis dans ces deux paragraphes.

4.3.1 Le cas où $\mathscr{B}$ est de type $I$. Il existe alors un polyrayon $k$-libre $\mathbf{r}$, de longueur $l$, tel que $\mathscr{B}$ soit une extension finie de $k_{\mathbf{r}}$, munie de l'unique valeur absolue prolongeant celle de $k_{\mathbf{r}}$; l'espace $\mathscr{M}(\mathscr{B})$ est réduit à $\{x\}$ (puisque c'est un singleton), et $\mathscr{B} \rightarrow \mathscr{H}(x)$ est un isomorphisme d'algèbres normées; le point $x$ est isolé dans $\left(\varphi^{a n}\right)^{-1}(O)$. Soit $\left(\tau_{1}, \ldots, \tau_{l}\right)$ une famille d'éléments de $\mathscr{A}$ inversibles en $x$ telle que le polyrayon $\left(\left|\tau_{i}(x)\right|\right)_{i}$ soit $k$-libre; remarquons qu'il existe une telle famille puisque $\mathscr{A} \rightarrow \mathscr{H}(x) \simeq \mathscr{B}$ est surjective.

Soit $\psi$ le morphisme $\mathscr{M}(\mathscr{A}) \rightarrow \mathbb{A}_{k}^{n, a n}$ correspondant à $\left(f_{1}, \ldots, f_{\delta}, \tau_{1}, \ldots, \tau_{l}\right)$. Par construction, $\psi(x)$ est l'unique point $t$ de $\mathbb{A}_{k}^{n, a n}$ tel que $T_{i}(t)$ soit nul pour tout $i$ compris entre 1 et $\delta$, et tel que $\left|T_{\delta+i}(t)\right|=\left|\tau_{i}(x)\right|$ pour tout $i$ compris entre 1 et $l$; le corps $\mathscr{H}(t)$ est isomorphe à $k_{\left(\left|\tau_{1}(x)\right|, \ldots,\left|\tau_{l}(x)\right|\right)}$. Le point $x$, étant isolé dans $\left(\varphi^{a n}\right)^{-1}(O)$, l'est a fortiori dans $\psi^{-1}(t)$ qui en est un sous-ensemble; par le lemme $1.21, \operatorname{dim}_{x} \psi=d(\mathscr{H}(x) / \mathscr{H}(t))$. Or $d(\mathscr{H}(x) / k)=l$, et $d(\mathscr{H}(t) / k)$ vaut également $l$, puisque $\left(\left|\tau_{i}(x)\right|\right)_{i}$ est $k$-libre. On en déduit que $d(\mathscr{H}(x) / \mathscr{H}(t))=0$; la famille $\left(f_{1}, \ldots, f_{\delta}, \tau_{1}, \ldots, \tau_{l}\right)$ est donc un système de paramètres $k$-analytiques en $x$.

4.3.2 Le cas où $\mathscr{B}$ est de type II. Il existe alors deux réels $r$ et $s$ tels que $0<r \leqslant s<1$ et tels

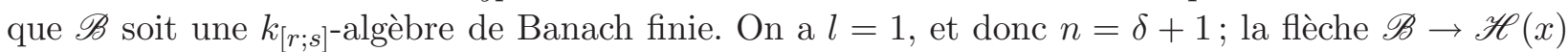
est un isomorphisme de corps topologiques. Soit $\tau$ un élément de $\mathscr{A}$ inversible en $x$ tel que $|\tau(x)|$ soit $k$-libre (autrement dit différent de 1) ; remarquons qu'il existe un tel $\tau$, puisque $\left|\mathscr{H}(x)^{*}\right| \neq\{1\}$ et puisque $\mathscr{A} \rightarrow \mathscr{H}(x) \simeq \mathscr{B}$ est surjective; l'application $y \mapsto|\tau(y)|$ induit un homéomorphisme entre $\mathscr{M}(\mathscr{B})$ et un intervalle compact de $\mathbb{R}_{+}^{*}$.

Soit $\psi$ le morphisme $\mathscr{M}(\mathscr{A}) \rightarrow \mathbb{A}_{k}^{n, a n}$ correspondant à $\left(f_{1}, \ldots, f_{\delta}, \tau\right)$. Par construction, $\psi(x)$ est l'unique point $t$ de $\mathbb{A}_{k}^{n, a n}$ tel que $T_{i}(t)$ soit nul pour tout $i$ compris entre 1 et $\delta$, et tel que $\left|T_{n}(t)\right|=|\tau(x)| ;$ le corps $\mathscr{H}(t)$ est isomorphe à $k_{|\tau(x)|}$. Comme $\mathscr{M}(\mathscr{B})$ est une composante connexe de $\left(\varphi^{a n}\right)^{-1}(O)$, et comme $|\tau|_{\mid \mathscr{M}(\mathscr{B})}$ est injective, le point $x$ est isolé dans $\psi^{-1}(t)$; par le lemme 1.21 , 


\section{VARIATION DE LA DIMENSION RELATIVE}

$\operatorname{dim}_{x} \psi=d(\mathscr{H}(x) / \mathscr{H}(t))$. Or $d(\mathscr{H}(x) / k)=1$, et $d(\mathscr{H}(t) / k)$ vaut également 1 , puisque $|\tau(x)|$ est $k$-libre. On en déduit que $d(\mathscr{H}(x) / \mathscr{H}(t))=0$; la famille $\left(f_{1}, \ldots, f_{\delta}, \tau\right)$ est donc un système de paramètres $k$-analytiques en $x$.

4.3.3 Remarque Dans chacun des deux cas considérés, le corps $\mathscr{H}(x)$ est une extension finie de $k_{\mathrm{r}}$ pour un certain polyrayon $k$-libre $\mathbf{r}$ de longueur $l$, et $\mathscr{A} \rightarrow \mathscr{H}(x)$ est surjective.

Définition 4.4. Une partie $\mathscr{P}$ d'une algèbre de Banach $\mathscr{B}$ sera dite presque dense si l'ensemble des éléments de $\mathscr{B}$ de la forme $f / g$, avec $f$ dans $\mathscr{P}$ et $g$ dans $\mathscr{P} \cap \mathscr{B}^{*}$, est dense dans $\mathscr{B}$ pour la semi-norme spectrale.

Lemme 4.5. Soit $\mathscr{A}$ une algèbre $k$-affinoïde non nulle et soit $\mathscr{X}$ son spectre. Pour toute partie presque dense $\mathscr{P}$ de $\mathscr{A}$, il existe un point fermé $\mathbf{x}$ de $\mathscr{X}$, un point $x$ de $\mathscr{M}(\mathscr{A})$ situé au-dessus de $\mathbf{x}$, et un système de paramètres $k$-analytiques en $x$ qui est formé d'éléments de $\mathscr{P}$.

Démonstration. Puisque la presque densité est une propriété relative à la semi-norme spectrale, elle est insensible aux phénomènes de nilpotence; il en va de même de la notion de système de paramètres analytiques. Ceci autorise à raisonner par récurrence noethérienne, et donc à supposer que tout fermé de Zariski strict et non vide de $\mathscr{X}$, quelque soit la structure de sous-schéma dont on le munit, satisfait le lemme. Soit $\mathscr{P}$ une partie presque dense de $\mathscr{A}$.

(i) Supposons que $\mathscr{X}$ soit de dimension de Krull nulle. Choisissons $x$ sur $\mathscr{M}(\mathscr{A})$. Le corps $\mathscr{H}(x)$ est alors, d'après la remarque 4.3.3, une extension finie de $k_{\mathrm{r}}$ pour un certain polyrayon mince et $k$-libre $\mathbf{r}$ dont on notera $l$ la longueur, et $\mathscr{A} \rightarrow \mathscr{H}(x)$ est surjective. Par presque densité de $\mathscr{P}$ dans $\mathscr{A}$, il existe un $l$-uplet $\left(p_{1}, \ldots, p_{l}\right)$ d'éléments de $\mathscr{P}$ tels que $\left(\left|p_{1}(x)\right|, \ldots,\left|p_{l}(x)\right|\right)$ soit $k$-libre; la famille $\left(p_{1}, \ldots, p_{l}\right)$ est alors un système de paramètres $k$-analytiques en $x$ par le 4.3 , et le lemme est démontré.

(ii) Supposons maintenant que $\mathscr{X}$ est de dimension de Krull strictement positive. Il existe donc dans $\mathscr{A}$ un idéal maximal qui n'est contenu dans aucun idéal premier minimal. On peut en conséquence trouver $f$ appartenant à $\mathscr{A}$ qui n'est pas inversible et dont le lieu des zéros ne contient aucune composante irréductible de $\mathscr{X}$.

Puisque $\mathscr{P}$ est presque dense, il existe une suite $\left(p_{n}\right)$ d'éléments de $\mathscr{P}$, et une suite $\left(q_{n}\right)$ d'éléments de $\mathscr{P} \cap \mathscr{A}^{*}$, telles que $p_{n} / q_{n}$ converge uniformément vers $f$. On déduit du lemme 2.1 qu'il n'y a qu'un nombre fini de $p_{n}$ qui sont inversibles; il est par ailleurs clair qu'il n'y a qu'un nombre fini de $p_{n}$ dont le lieu des zéros contient une composante irréductible de $\mathscr{X}$. Il existe en conséquence un élément $p$ de $\mathscr{P}$ dont le lieu des zéros $\mathscr{Y}$ est un fermé de Zariski strict et non vide de $\mathscr{X}$. On peut donc lui appliquer le lemme, ce qu'on va faire en considérant l'image (évidemment presque dense) de $\mathscr{P}$ dans $\mathscr{A} /(p)$.

On obtient l'existence d'un point fermé $\mathbf{x}$ de $\mathscr{Y}$, d'un point $x$ de $\mathscr{M}(\mathscr{A})$ situé au-dessus de $\mathbf{x}$ et d'un système de paramètres $k$-analytiques en $x$ vu comme point de $\mathscr{M}(\mathscr{A} /(p))$, qui est de la forme $\left(\overline{p_{1}}, \ldots, \overline{p_{r}}\right)$ où les $p_{i}$ appartiennent à $\mathscr{P} ;$ l'entier $r$ est égal à $\operatorname{dim}_{k, x} \mathscr{M}(\mathscr{A} /(p))$.

Soit $\varphi$ (respectivement $\psi$ ) la flèche de $\mathscr{M}(\mathscr{A})$ vers $\mathbb{A}_{k}^{r, a n}$ (respectivement $\mathbb{A}_{k}^{r+1, a n}$ ) définie par $\left(p_{1}, \ldots, p_{r}\right)$ (respectivement par $\left.\left(p, p_{1}, \ldots, p_{r}\right)\right)$. Soit $z$ (respectivement $t$ ) l'image de $x$ par $\varphi$ (respectivement $\psi$ ). Par choix des $p_{i}$, on a $\operatorname{dim}_{x} \varphi_{\mid \mathscr{M}(\mathscr{A} /(p))}=0$. Soit $\iota$ l'immersion fermée $\left(0\right.$, Id) de $\mathbb{A}_{k}^{r, a n}$ dans $\mathbb{A}_{k}^{r+1, a n} \simeq \mathbb{A}_{k}^{1, a n} \times \mathbb{A}_{k}^{r, a n}$. Comme $p$ s'annule en $x$, on a $t=\iota(z) ;$ en conséquence $\mathscr{H}(z)=\mathscr{H}(t)$, et la fibre $\psi^{-1}(t)$ coïncide comme espace topologique avec $\left(\varphi_{\mid \mathscr{M}(\mathscr{A} /(p))}\right)^{-1}(z)$. Dès lors

$$
\operatorname{dim}_{\mathscr{H}(t), x} \psi^{-1}(t)=\operatorname{dim}_{\mathscr{H}(z), x}(\varphi \mid \mathscr{M}(\mathscr{A} /(p)))^{-1}(z)=\operatorname{dim}_{x} \varphi_{\mid \mathscr{M}(\mathscr{A} /(p))}=0 .
$$

Soit $Z$ une composante irréductible de $\mathscr{M}(\mathscr{A})$ contenant $x$; la restriction de $p$ à $Z$ n'est pas nulle (puisque le lieu des zéros de $p$ ne contient pas $Z$ ) ni inversible (puisque $p(x)=0$ ). Soit $Z^{\prime}$ une 


\section{A. Ducros}

composante irréductible de $\mathscr{M}(\mathscr{A} /(p))$ contenant $x$ et incluse dans $Z$. Comme $\operatorname{codim}_{\mathrm{Krull}}\left(Z^{\prime}, Z\right)=1$ grâce au Hauptidealsatz, $\operatorname{dim}_{k} Z^{\prime}=\operatorname{dim}_{k} Z-1$, et $\operatorname{donc}_{\operatorname{dim}_{k, x}} \mathscr{M}(\mathscr{A})=\operatorname{dim}_{k, x} \mathscr{M}(\mathscr{A} /(p))+1=$ $r+1$; les $r+1$ éléments $p, p_{1}, \ldots, p_{r}$ de $\mathscr{P}$ constituent dès lors un système de paramètres $k$-analytiques en $x$ vu comme point de $\mathscr{M}(\mathscr{A})$.

\section{Le théorème de factorisation}

ThÉorème 4.6. Soit $\varphi: Y \rightarrow X$ un morphisme entre espaces $k$-affinoïdes. Soit y un point de $Y$; notons $n$ l'entier $\operatorname{dim}_{y} \varphi$. Le morphisme $\varphi$ se factorise par une flèche $\psi$ de $Y$ vers $\mathbb{A}_{X}^{n}$ telle que $\operatorname{dim}_{y} \psi$ soit nulle.

Démonstration. Soit $x$ l'image de $y$ sur $X$. On note $\mathscr{A}$ (respectivement $\mathscr{A}_{x}$ ) l'algèbre des fonctions sur $Y$ (respectivement $Y_{x}$ ). L'image dans $\mathscr{A}_{x}$ de la limite inductive des $\mathscr{O}_{Y}\left(\varphi^{-1}(U)\right.$ ), où $U$ parcourt l'ensemble des voisinages affinoïdes de $x$ dans $X$, est dense; comme $x$ possède une base de voisinages rationnels dans $X$, l'image de $\mathscr{A}$ dans $\mathscr{A}_{x}$ est presque dense. Soit $F$ l'adhérence de Zariski de $\{y\}$ dans $Y$, et soit $\mathscr{I}$ un idéal de $\mathscr{A}$ définissant $F$. Soit $H$ une composante irréductible de $F \cap Y_{x}$ contenant $y$. Soit $l$ la dimension $\mathscr{H}(x)$-analytique de $H$, et soit $\delta$ sa codimension de Krull dans $Y_{x}$; on a $\delta+l \leqslant n$.

Il existe $\delta$ fonctions $f_{1}, \ldots, f_{\delta}$ appartenant à $\mathscr{I}$ telles que $H$ soit une composante irréductible du lieu des zéros $H^{\prime}$ des $f_{i \mid Y_{x}}$. En effet, c'est évident si $\delta=0$. Si $\delta>0$, il existe (par exemple par le lemme de sélection des idéaux premiers) un élément $f$ de $\mathscr{I} \mathscr{A}_{x}$ dont le lieu des zéros ne contient aucune composante irréductible $T$ de $Y_{x}$ vérifiant $H \subset T$ et $\operatorname{codim}_{\mathrm{Krull}}(H, T)=\delta$. Grâce à la presque densité de l'image de $\mathscr{A}$ dans $\mathscr{A}_{x}$, on peut de surcroît supposer que $f$ est la restriction à $Y_{x}$ d'une fonction $f_{1}$ de $\mathscr{I}$. Si $Z$ désigne le fermé de Zariski de $Y$ d'équation $f_{1}=0$, la codimension de $H$ dans $Z_{x}$ est égale à $\delta-1$ en vertu du Hauptidealsatz, et l'on conclut par récurrence.

Soit $H_{0}$ l'ouvert de Zariski de $H$ formé des points qui ne sont situés sur aucune autre composante irréductible de $H^{\prime}$; c'est un domaine $\mathscr{H}(x)$-analytique non vide de $H$, il contient donc un domaine rationnel non vide $H_{1}$ de $H$. Comme $H$ est irréductible, $\operatorname{dim}_{\mathscr{H}(x)} H_{1}=l$. L'image de $\mathscr{A}_{x}$ dans l'anneau des fonctions de $H_{1}$ est presque dense; l'image de $\mathscr{A}$ dans $\mathscr{A}_{x}$ étant également presque dense, l'image de $\mathscr{A}$ dans l'anneau des fonctions de $H_{1}$ est presque dense. Par le lemme 4.5, il existe $h \in H$ et $l$ fonctions $g_{1}, \ldots, g_{l}$ appartenant à $\mathscr{A}$ telles que $\operatorname{dim}_{h} \gamma_{\mid H_{1}}=0$, où $\gamma$ est le $X$-morphisme de $Y$ vers $\mathbb{A}_{X}^{l}$ induit par les $g_{i}$. La remarque 1.20 assure que $\operatorname{dim}_{h} \gamma_{\mid H}=0$; cela signifie que $\mathscr{H}(h)$ est fini sur $\mathscr{H}(\gamma(h))$ et que $h$ est isolé dans $\left(\gamma_{\mid H}\right)^{-1}(\gamma(h))$ (lemme 1.21). Soit $\psi$ le $X$-morphisme de $Y$ vers $\mathbb{A}_{X}^{\delta+l}$ induit par $\left(f_{1}, \ldots, f_{\delta}, g_{1}, \ldots, g_{l}\right)$. Chaque $f_{i}$ s'annule sur $H$. On en déduit que $\mathscr{H}(\psi(h))=\mathscr{H}(\gamma(h))$, et donc que $\mathscr{H}(h)$ est fini sur $\mathscr{H}(\psi(h))$. D'autre part, il est clair que $\psi^{-1}(\psi(h))=\gamma^{-1}(\gamma(h)) \cap H^{\prime}$; or $H$ est par construction l'unique composante irréductible de $H^{\prime}$ contenant $h$; il s'ensuit que $h$ est isolé dans $\psi^{-1}(\psi(h))$. Grâce au lemme 1.21, on conclut que $\operatorname{dim}_{h} \psi=0$.

Le sous-ensemble de $Y$ formé des points $z$ tels que $\operatorname{dim}_{z} \psi$ soit nulle en est un ouvert de Zariski par la proposition 3.5. Il contient $h$ grâce à ce qui précède; or $h$ est situé sur $F$, qui est l'adhérence de Zariski de $\{y\}$ dans $Y$. En conséquence $\operatorname{dim}_{y} \psi$ vaut également zéro. Par le 1.25 , on a alors $n \leqslant \delta+l$; on a vu d'autre part que $\delta+l \leqslant n$, d'où l'égalité $\delta+l=n$. La démonstration est terminée.

À l'aide de la proposition 3.5, on obtient en corollaire une variante analytique d'un théorème de factorisation de Raynaud et Gruson [RG71, théorème 1.1.1], dont nous espérons qu'elle sera utile pour l'étude de la platitude dans le contexte des espaces de Berkovich.

Corollaire 4.7. Soit $\varphi$ un morphisme entre deux bons espaces $k$-analytiques $Y$ et $X$, soit $y$ un point de $Y$ et soit $n$ la dimension de $\varphi$ en $y$. Il existe un voisinage affinoïde $V$ de $y$ dans $Y$, un 


\section{VARIATION DE LA DIMENSION RELATIVE}

$X$-espace lisse $T$ purement de dimension $k$-analytique $n$, et un domaine affinoïde $W$ de $T$, tels que $\varphi_{\mid V}$ se factorise par une flèche finie $V \rightarrow W$.

Remarque 4.8. Ce corollaire assure l'existence localement sur $Y$ d'une factorisation agréable. Supposons que $\left|k^{*}\right| \neq\{1\}$ et que $X$ et $Y$ sont strictement $k$-analytiques, et retirons l'hypothèse qu'ils sont bons. En recourant à la géométrie formelle, Bosch et Lütkebohmert ont établi sous ces conditions [BL93, Theorem 1.5] l'existence G-localement sur $Y$ d'une telle factorisation; cela en fournit une au voisinage de tout point $y$ de $Y$ tel que $\widetilde{\mathscr{H}(y)} / \widetilde{k}$ soit algébrique, et en particulier au voisinage de tout point rigide; mais pour y quelconque, cela entraîne simplement l'existence d'un nombre fini de domaines affinoïdes dont la réunion est un voisinage de $y$, et sur chacun desquels on peut trouver une factorisation du type souhaité, sans possibilité de recollement a priori.

\section{Le théorème de semi-continuité}

ThÉorème 4.9. Soit $\varphi: Y \rightarrow X$ un morphisme d'espaces $k$-analytiques. La fonction $y \mapsto \operatorname{dim}_{y} \varphi$ est semi-continue supérieurement pour la topologie de Zariski sur $Y$.

Démonstration. La question est locale pour la G-topologie, ce qui autorise à supposer $Y$ et $X$ affinoïdes. Par récurrence noethérienne, on peut supposer que la restriction de $\varphi$ à chacun des fermés de Zariski stricts de $Y$ satisfait les conclusions du corollaire. Si $Y$ est vide, il n'y a rien à démontrer; sinon, soit $y$ un point de $Y$ en lequel la dimension de $\varphi$ atteint son minimum, que l'on notera $d$. Par le théorème 4.6 ci-dessus, il existe une factorisation de $\varphi$ par un morphisme $\psi: Y \rightarrow \mathbb{A}_{X}^{d}$ tel que $\operatorname{dim}_{y} \psi$ soit nulle. La proposition 3.5 affirme que l'ensemble $U$ des points $z$ de $Y$ en lesquels on a l'égalité $\operatorname{dim}_{z} \psi=0$ est un ouvert de Zariski; il est non vide puisqu'il contient $y$; soit $F$ son fermé complémentaire. Par minimalité de $d$ et d'après le $1.25, \operatorname{dim}_{z} \varphi=d$ pour tout $z$ de $U$. Soit $i$ un entier, et soit $F_{i}$ le lieu des points de $Y$ en lesquels la dimension de $\varphi$ est supérieure ou égale à $i$. Si $i \leqslant d$ alors $F_{i}$ est égal à $Y$ tout entier; si $i \geqslant d+1$ alors $F_{i}$ est inclus dans $F$, et coïncide en vertu du lemme 1.26 avec l'ensemble $F_{i}^{\prime}$ des points de $F$ en lesquels la dimension de $\varphi_{\mid F}$ est supérieure ou égale à $i$. Par l'hypothèse de récurrence, $F_{i}^{\prime}$ est un fermé de Zariski de $F$, et donc de $Y$. Tous les $F_{i}$ sont donc des fermés de Zariski de $Y$, ce qui achève la démonstration.

\section{REMERCIEMENTS}

Je sais gré au rapporteur d'avoir lu extrêmement attentivement une première version de ce texte, de m'avoir proposé de nombreuses modifications qui ont grandement contribué à l'améliorer, et de m'avoir incité à publier les résultats de base sur la théorie de la dimension et le Nullstellensatz affinoïde dont je me sers dans ce travail; ceux-ci n'étaient alors disponibles que sous forme de prépublication, ils font désormais l'objet des deux premiers chapitres de cet article.

\section{RÉFÉRENCES}

Ber90 V. Berkovich, Spectral theory and analytic geometry over non-archimedean fields, Mathematical Surveys and Monographs, vol. 33 (American Mathematical Society, Providence, RI, 1990).

Ber93 V. Berkovich, Étale cohomology for non-archimedean analytic spaces, Publ. Math. Inst. Hautes Études Sci. 78 (1993), 5-161.

Ber96 V. Berkovich, Vanishing cycles for non-Archimedean analytic spaces, J. Amer. Math. Soc. 9 (1996), 1187-1209.

Ber08 V. Berkovich, A non-Archimedean interpretation of the weight zero subspaces of limit mixed Hodge structures, in Algebra, arithmetic and geometry - Manin Festschrift (Birkhäuser, Boston, 2008), to appear. 


\section{VARIATION DE LA DIMENSION RELATIVE}

BGR84 S. Bosch, S. Güntzer and U. Remmert, Non-Archimedean analysis. A systematic approach to rigid analytic geometry, Grundlehren der Mathematischen Wissenschaften, vol. 261 (Springer, Berlin, 1984).

BL93 S. Bosch and W. Lütkebohmert, Formal and rigid geometry. II. Flattening techniques, Math. Ann. 296 (1993), 403-429.

Con99 B. Conrad, Irreducible components of rigid spaces, Ann. Inst. Fourier (Grenoble) 49 (1999), 473-541.

Con06 B. Conrad, Modular curves and rigid-analytic spaces, Pure Appl. Math. Q. 2 (2006), no. 1 (John Coates special issue, Part I).

Duc03 A. Ducros, Parties semi-algébriques d'une variété algébrique p-adique, Manuscripta Math. 111 (2003), 513-528.

Gru66 L. Gruson, Théorie de Fredholm p-adique, Bull. Soc. Math. France 94 (1966), 67-95.

Kie68 R. Kiehl, Analytische Familien affinoider Algebren, S.-B. Heidelberger Akad. Wiss. Math.-Natur. Kl. 1968 (1968), 23-49.

RG71 M. Raynaud and L. Gruson, Critères de platitude et de projectivité. Techniques de 'platification' d'un module, Invent. Math. 13 (1971), 1-89.

Tem04 M. Temkin, On local properties of non-Archimedean analytic spaces. II, Israel J. Math. 140 (2004), $1-27$.

Thu07 A. Thuillier, Géométrie toroïdale et géométrie analytique non archimédienne. Application au type d'homotopie de certains schémas formels, Manuscripta Math. 123 (2007), 381-451.

Antoine Ducros ducros@unice.fr

Laboratoire J.-A. Dieudonné, Université de Nice - Sophia Antipolis, Parc Valrose, 06108 Nice cedex 02, France 